\title{
SYMPLECTIC GEOMETRIES ON $T^{*} \widetilde{G}$, HAMILTONIAN GROUP ACTIONS AND INTEGRABLE SYSTEMS ${ }^{\dagger}$
}

\author{
J. HARnAD ${ }^{1}$ AND B.A. KupershmidT ${ }^{2}$
}

\begin{abstract}
Various Hamiltonian actions of loop groups $\widetilde{G}$ and of the algebra diff ${ }_{1}$ of first order differential operators in one variable are defined on the cotangent bundle $T^{*} \widetilde{G}$. The moment maps generating the $\operatorname{diff}_{1}$ actions are shown to factorize through those generating the loop group actions, thereby defining commuting diagrams of Poisson maps to the duals of the corresponding centrally extended algebras. The maps are then used to derive a number of infinite commuting families of Hamiltonian flows that are nonabelian generalizations of the dispersive water wave hierarchies. As a further application, sets of pairs of generators of the nonabelian $\mathrm{mKdV}$ hierarchies are shown to give a commuting hierarchy on $T^{*} \widetilde{G}$ that contain the WZW system as its first element.
\end{abstract}

\section{Introduction}

Integrable $1+1$-dimensional systems have long been recognized as closely connected with loop algebras (see e.g. [AHP, DS, RS, FNR]). The rôle played by loop groups in the Hamiltonian setting is in a sense more fundamental, but also more subtle [DJMK, SW, RS, W1, W2, H, HK1]. For many integrable systems the underlying phase space may be taken as the cotangent bundle $T^{*} L G$ of a loop group $L G$. However, the symplectic structure is not necessarily the canonical one; it may more generally be a member of a 1-parameter family obtained by shifting the canonical form by a multiple of the 2-cocycle on the corresponding loop algebra $L \mathfrak{g}$ associated to an

$\dagger$ Research supported in part by the Natural Sciences and Engineering Research Council of Canada and the National Science Foundation.

${ }^{1}$ Department of Mathematics and Statistics, Concordia University, Montréal, P.Q. and Centre de Recherches Mathématiques, Université de Montréal, C.P. 6128-A, Montréal, P.Q. H3C 3J7

2 The University of Tennessee Space Institute, Tullahoma, TN 37388, U.S.A. 
$A d$-invariant metric on $\mathfrak{g}$. It is this shift that leads to a phase space structure involving the centrally extended loop algebra. Reduction of $T^{*} L G$ under left or right translations leads to the space $L G / G=\left\{\right.$ Maps $\left.S^{1} \rightarrow G\right\} /\{$ constant loops $\}$ (a coadjoint orbit in the dual $L \mathfrak{g}^{\wedge *}$ of the centrally extended loop algebra), with the natural symplectic form related to the 2-cocycle (cf. [PS, H]). By combining such reductions with the moment maps generating the natural action of diff $S^{1}$ on $L G$ and $L \mathfrak{g}$, one obtains Poisson maps relating integrable hierarchies of the $\mathrm{KdV}$ and nonabelian $\mathrm{mKdV}$ type $[\mathbf{K u 2}, \mathbf{H K} \mathbf{1}$, HK2]. In this context, the moment map provides a nonabelian generalization of the well known Miura map.

In the present work we show, using the moment maps generating certain infinitesimal Hamiltonian actions of the algebra diff 1 of first order differential operators in one variable on the nonreduced phase space $T^{*} L G$, how some new, nonstandard Lax equations determining infinite commuting families of flows are derived. These systems are related to the integrable hierarchy of dispersive water wave (DWW) equations [Ku1] in a way analogous to the relation between the nonabelian modified $\mathrm{KdV}$ and $\mathrm{KdV}$ hierarchies. They will be referred to here as the $\mathfrak{g}$-mDWW (g-modified DWW) systems. The relevant phase space for the latter is the dual $(L \mathfrak{g} \oplus L \mathfrak{g})^{\wedge *}$ of the centrally extended direct sum of two copies of the loop algebra $L \mathfrak{g}$. (Alternatively, nonperiodic, rapidly decreasing boundary conditions may be allowed, in which case the corresponding groups and algebras will denoted $\widetilde{G}$ and $\widetilde{\mathfrak{g}}$, respectively.) A second version, involving a different Hamiltonian action of $\operatorname{diff}_{1}$ on $T^{*} L G$, leads to systems on the phase space $\left(L \mathfrak{g}+L \mathfrak{g}_{A}\right)^{*}$ (or $\left.(\widetilde{\mathfrak{g}}+\widetilde{\mathfrak{g}})^{\wedge *}\right)$, where the subscript $A$ denotes abelianization, and the sum is semi-direct. These will be referred to as the $\mathfrak{g}-\mathrm{m}^{2} \mathrm{DWW}$ (second $\mathfrak{g}$-modified DWW) systems.

The relevant family of symplectic structures on $T^{*} L G$ (or $T^{*} \widetilde{G}$ ) is defined in Section 1a. The rest of Section 1 is devoted to a systematic study of the various Hamiltonian actions of $\widetilde{G}$ on $T^{*} \widetilde{G}$, $(\widetilde{\mathfrak{g}} \oplus \widetilde{\mathfrak{g}})^{\wedge *}$ and $\left(\widetilde{\mathfrak{g}}+\widetilde{\mathfrak{g}}_{A}\right)^{\wedge *}$, as well as the that of the group $\mathcal{D}_{1} \ltimes \mathcal{D}_{0}$ corresponding to diff 1 . The associated moment maps are derived and shown to form commuting triplets of Poisson maps into $(\widetilde{\mathfrak{g}} \oplus \widetilde{\mathfrak{g}})^{\wedge *}\left(\right.$ or $\left.\left(\widetilde{\mathfrak{g}}+\widetilde{\mathfrak{g}}_{A}\right)^{\wedge *}\right)$ and $\operatorname{diff}_{1}^{\wedge *}$, where $\operatorname{diff}_{1} \wedge$ is a member of a 3-parameter family of central extensions of the algebra diff 1 . Using these Poisson maps in a way analogous to the generalized Miura map, various integrable systems associated with the dispersive water wave hierarchy 
are constructed in Section 2. Since the KdV and nonabelian mKdV hierarchies may be recovered by restricting every second flow to a certain invariant manifold, the DWW and $\mathfrak{g}-\mathrm{mDWW}$ systems may be viewed as generalizations of the KdV and nonabelian mKdV systems, which are more usually defined on the phase spaces (diff $\left.S^{1}\right)^{\wedge *}$ and $\widetilde{\mathfrak{g}}^{\wedge *}$ (or $L \mathfrak{g}^{\wedge *}$ ), respectively. The Poisson maps constructed in Section 1 are recast in Section 2 in the language of differential algebras, Hamiltonian matrices and Hamiltonian maps. In addition to the DWW, $\mathfrak{g}-\mathrm{mDWW}$ and $\mathfrak{g}-\mathrm{m}^{2} \mathrm{DWW}$ hierarchies, we also obtain integrable hierarchies on $T^{*} L G$ via pullbacks under the appropriate Hamiltonian maps. As a further application of these results, we show that there exists a natural notion of higher WZW systems associated to pairs of KdV systems that are related to the separated left and right translational modes of the WZW system.

\section{Hamiltonian Group Actions on $T^{*} \widetilde{G}$}

\section{1a. Symplectic Structures on $T^{*} \widetilde{G}$}

Let $G$ be a Lie group with Lie algebra $\mathfrak{g}$ on which an Ad-invariant scalar product $\mathcal{B}: \mathfrak{g} \times \mathfrak{g} \rightarrow \mathbb{R}$ is defined. We denote by $\widetilde{G}$ the group of smooth maps $g: \mathbb{R} \rightarrow G$, with pointwise multiplication, and by $\widetilde{\mathfrak{g}}$ its Lie algebra, consisting similarly of maps $X: \mathbb{R} \rightarrow \mathfrak{g}$. If the periodicity conditions $g(\sigma+2 \pi)=g(\sigma), X(\sigma+2 \pi)=X(\sigma)$ are added, $\widetilde{G}$ and $\widetilde{\mathfrak{g}}$ become the loop group $L G$ and loop algebra $L \mathfrak{g}$, respectively. For the nonperiodic case we also require $g \in \widetilde{G}, X \in \widetilde{\mathfrak{g}}$ to satisfy $L^{2}$ boundary conditions such that the integral

$$
\begin{aligned}
\langle X, Y\rangle & :=\int \mathcal{B}(X(\sigma), Y(\sigma)) d \sigma \\
X, Y & \in \widetilde{\mathfrak{g}}
\end{aligned}
$$

converge over $\mathbb{R}$, defining an Ad-invariant scalar product on $\widetilde{\mathfrak{g}}$, and the same integral converge when the pair $(X, Y)$ is replaced by $\left(g^{\prime} g^{-1}, h^{\prime} h^{-1}\right)$ for any pair of group elements $g, h \in \widetilde{G}$. For $L \mathfrak{g}$ the integral in (1.1) is understood as evaluated over a period.

The scalar product $\mathcal{B}$ gives an identification between $\mathfrak{g}$ and its dual space $\mathfrak{g}^{*}$, while $<,>$ gives an identification of $\widetilde{\mathfrak{g}}$ as a dense subspace of $\widetilde{\mathfrak{g}}^{*}$. For our purposes, when 
speaking of $\widetilde{\mathfrak{g}}^{*}$, we shall really only mean elements in this dense subspace, and hence the same notation

$$
\begin{aligned}
& \langle U, X\rangle:=\int \mathcal{B}(U(\sigma), X(\sigma)) d \sigma \\
& U \in \widetilde{\mathfrak{g}}^{*}, \quad X \in \widetilde{\mathfrak{g}}
\end{aligned}
$$

will be used to denote the dual pairing $\widetilde{\mathfrak{g}}^{*} \times \widetilde{\mathfrak{g}} \rightarrow \mathbb{R}$. The 2-cocycle

$$
\begin{aligned}
c: \widetilde{\mathfrak{g}} \times \widetilde{\mathfrak{g}} & \rightarrow \mathbb{R} \\
c(X, Y) & :=\left\langle X, Y^{\prime}\right\rangle, \\
Y^{\prime} & :=\frac{d Y}{d \sigma},
\end{aligned}
$$

is used to define the centrally extended algebra, denoted $\widetilde{\mathfrak{g}}^{\wedge}$ (or $L \mathfrak{g}^{\wedge}$ ), identified as the space $\widetilde{\mathfrak{g}}+\mathbb{R}($ respectively $L \mathfrak{g}+\mathbb{R})$, with Lie brackets:

$$
\begin{gathered}
{[(X, a),(Y, b)]:=\left([X, Y],\left\langle X, Y^{\prime}\right\rangle\right)} \\
X, Y \in \widetilde{\mathfrak{g}}, \quad a, b \in \mathbb{R} .
\end{gathered}
$$

The dual space $\widetilde{\mathfrak{g}}^{\wedge *}$ is again identified with $\widetilde{\mathfrak{g}}^{*}+\mathbb{R} \sim \widetilde{\mathfrak{g}}+\mathbb{R}$, with dual pairing

$$
\begin{gathered}
\langle(U, a),(X, b)\rangle:=\langle U, X\rangle+a b \\
(U, a) \in \widetilde{\mathfrak{g}}^{\wedge *}, \quad(X, b) \in \widetilde{\mathfrak{g}}^{\wedge} .
\end{gathered}
$$

Denoting by $a d_{X}^{*}$ and $A d_{g}^{*}$ the coadjoint representations of elements $X \in \widetilde{\mathfrak{g}}, g \in \widetilde{G}$ in the algebra and group, respectively, the extended coadjoint representation on $\widetilde{\mathfrak{g}}^{\wedge *}$ is given by the formulae:

$$
\begin{aligned}
\widehat{a d}_{(X, a)}^{*}(U, b) & =\left(\operatorname{ad}_{X}^{*} U+b X^{\prime}, 0\right) \\
\widehat{A d}_{\hat{g}}^{*}(U, b) & =\left(\operatorname{Ad}_{g}^{*} U+b g^{\prime} g^{-1}, b\right)
\end{aligned}
$$

Although the centrally extended group $\widetilde{G}^{\wedge} \rightarrow \widetilde{G}$ with Lie algebra $\widetilde{\mathfrak{g}}^{\wedge}$ is a nontrivial line or circle bundle over $\widetilde{G}$ (cf. [PS]), its action on $\widetilde{\mathfrak{g}}^{\wedge *}$ depends only on the projection of elements $\hat{g} \in \widetilde{G}^{\wedge}$ to their image $g \in \widetilde{G}$, as indicated in (1.6b).

From $(1.4),(1.5)$, the Lie Poisson bracket on $\widetilde{\mathfrak{g}}^{\wedge *}$ is determined by the formula

$$
\left.\{\langle\cdot \mid(X, a)\rangle,\langle\cdot \mid(Y, b)\rangle\}\right|_{(U, e)}=\langle U,[X, Y]\rangle+e c(X, Y)
$$


where $\langle\cdot \mid(X, a)\rangle$ denotes the linear functional on $\tilde{\mathfrak{g}}^{\wedge *}$ with value $\langle(U, e),(X, a)\rangle$ at $(U, e) \in \widetilde{\mathfrak{g}}^{\wedge *}$, corresponding to pairing with the element $(X, a) \in \widetilde{\mathfrak{g}}^{\wedge}$.

Now consider the cotangent bundle $T^{*} \widetilde{G}$, identified through left translations with the product $\widetilde{G} \times \widetilde{\mathfrak{g}}^{*}$. Denoting a typical element by the pair $\left(g \in \widetilde{G}, \mu \in \widetilde{\mathfrak{g}}^{*}\right)$, the canonical symplectic form on $T^{*} \widetilde{G}$ may be expressed as

$$
\omega_{0}=-\delta\left\langle\mu, g^{-1} \delta g\right\rangle
$$

where $\delta$ denotes the functional differential and $g^{-1} \delta g$ is the left invariant Maurer-Cartan form on $\widetilde{G}$. In the following, a more general family of symplectic forms will be needed (cf. $[\mathbf{H}]$ ), defined by the formula:

$$
\omega_{k}:=-\delta\left\langle\mu, g^{-1} \delta g\right\rangle+\frac{k}{2}\left\langle\delta\left(g^{-1} g^{\prime}\right) \wedge g^{-1} \delta g\right\rangle
$$

where $k$ is a real parameter. Here, $g^{-1} g^{\prime}$ is viewed as the element of $\widetilde{\mathfrak{g}}$ whose value at $\sigma \in S^{1}$ is obtained by left translation of the tangent vector $g^{\prime}(\sigma) \in T_{g(\sigma)} G$ to the identity $e \in G$. It is shown in $[\mathbf{H}]$ that $\omega_{k}$ is closed and weakly nondegenerate. The corresponding Poisson brackets are defined by the formulae (cf. FT]):

$$
\begin{aligned}
\left\{f_{1}, f_{2}\right\} & =0 \\
\left.\{f,\langle\cdot \mid X\rangle\}\right|_{(g, \mu)} & =\int \delta f(g X) d \sigma \\
\left.\{\langle\cdot \mid X\rangle,\langle\cdot \mid Y\rangle\}\right|_{(g, \mu)} & =-\left\langle\mu+k g^{-1} g^{\prime},[X, Y]\right\rangle+k c(X, Y), \\
X, Y & \in \widetilde{\mathfrak{g}}
\end{aligned}
$$

where $f, f_{1}, f_{2}$ are functionals on $\widetilde{G} \times \widetilde{\mathfrak{g}}^{*}$ depending only on the first factor $g \in \widetilde{G},\langle. \mid X\rangle$ is the linear form on the second factor corresponding to the algebra element $X \in \tilde{\mathfrak{g}}$ and the RHS of (1.10b) signifies evaluation of the functional differential $\delta f$ on the vector $g X \in T_{g} \widetilde{G}$ obtained by left translation of $X \in \widetilde{\mathfrak{g}} \sim T_{e} \widetilde{G}$ to $g \in \widetilde{G}$ (i.e. evaluation of the corresponding left invariant vector field on $f$ ). The Poisson brackets (1.10a-c) follow from the following formula for the Hamiltonian vector field $X_{H}$ of an arbitrary smooth functional $H$ defined over $T^{*} \widetilde{G}$ :

$$
X_{H}=\left\langle g \frac{\delta H}{\delta \mu}, \frac{\delta}{\delta g}\right\rangle+\left\langle\left[\mu+k g^{-1} g^{\prime}, \frac{\delta H}{\delta \mu}\right]+k\left(\frac{\delta H}{\delta \mu}\right)^{\prime}-\frac{\delta H}{\delta g} g, \frac{\delta}{\delta \mu}\right\rangle
$$


(so that $\left.\left.X_{H}\right\lrcorner \omega_{k}=\delta(H)=\left\langle\frac{\delta H}{\delta g}, \delta g\right\rangle+\left\langle\frac{\delta H}{\delta \mu}, \delta \mu\right\rangle\right)$.

1b. Hamiltonian $\widetilde{G} \times \widetilde{G}$ Action on $T^{*} \widetilde{G}$

The canonical lift of the right translation action of $\widetilde{G}$ to $T^{*} \widetilde{G}$ is given by:

$$
R_{h^{-1}}:(g, \mu) \longmapsto\left(g h^{-1}, h \mu h^{-1}\right)
$$

where the inverse $h^{-1}$ is used to make this a left action. This action leaves the symplectic form $\omega_{k}$ invariant for all $k$. To lift the left translation action so as to preserve $\omega_{k}$, it is necessary to modify the usual canonical lift by defining:

$$
L_{h}:(g, \mu) \longmapsto\left(h g, \mu+k g^{-1} h^{-1} h^{\prime} g\right)
$$

Substitution in eq. (1.9) shows that this does leave $\omega_{k}$ invariant and composing these maps shows that (1.12) defines a left action, commuting with the right translation action (1.11). The following result summarizes the Hamiltonian properties of these actions.

Proposition 1.1. The $R$ and $L$ actions (1.11), (1.12) are generated in terms of Hamiltonian flows by the moment maps:

$$
\begin{aligned}
& J^{L}: T^{*} \widetilde{G} \longrightarrow \widetilde{\mathfrak{g}}^{*} \\
& J^{L}:(g, \mu) \longmapsto g \mu g^{-1} \\
& J_{k}^{R}: T^{*} \widetilde{G} \longrightarrow \widetilde{\mathfrak{g}}^{*} \\
& J_{k}^{R}:(g, \mu) \longmapsto-\mu+k g^{-1} g^{\prime},
\end{aligned}
$$

respectively. The functions obtained by pairing these maps with elements of $\widetilde{\mathfrak{g}}$ Poisson commute with each other, but the maps are nonequivariant, having as 2-cocycles kc and $-k c$, respectively. Thus, the Hamiltonian generators

$$
J_{X}^{L}:=\left\langle J^{L}, X\right\rangle, \quad J_{X}^{R}:=\left\langle J_{k}^{R}, X\right\rangle
$$

satisfy the following Poisson bracket relations:

$$
\begin{aligned}
& \left\{J_{X}^{L}, J_{Y}^{L}\right\}=J_{[X, Y]}^{L}+k c(X, Y) \\
& \left\{J_{X}^{R}, J_{Y}^{R}\right\}=J_{[X, Y]}^{R}-k c(X, Y) \\
& \left\{J_{X}^{L}, J_{Y}^{R}\right\}=0 .
\end{aligned}
$$


Proof. Differentiating the actions (1.11), (1.12) along a 1-parameter subgroup $\{h(t)=$ $\exp (-t X)\}$, we find the following representations of $\widetilde{\mathfrak{g}}$ in terms of functional vector fields on $T^{*} \widetilde{G}$ :

$$
\begin{gathered}
X_{X}^{R}=\left\langle g X, \frac{\delta}{\delta g}\right\rangle-\left\langle[X, \mu], \frac{\delta}{\delta \mu}\right\rangle \\
X_{X}^{L}=-\left\langle X g, \frac{\delta}{\delta g}\right\rangle-\left\langle k g^{-1} X^{\prime} g, \frac{\delta}{\delta \mu}\right\rangle,
\end{gathered}
$$

where the first terms $\left\langle g X, \frac{\delta}{\delta g}\right\rangle$ and $\left\langle X g, \frac{\delta}{\delta g}\right\rangle$ denote, respectively, the left and right invariant vector fields on the first factor $\widetilde{G}$ in $T^{*} \widetilde{G}=\widetilde{G} \times \widetilde{\mathfrak{g}}^{*}$, with value $X$ at the identity $e \in \widetilde{G}$, and the second terms, of the form $\left\langle\eta(g, \mu), \frac{\delta}{\delta \mu}\right\rangle$, denote the vector field on the second factor $\widetilde{\mathfrak{g}}^{*}$ having value $\eta(g, \mu) \in \widetilde{\mathfrak{g}}^{*}$ at the point $(g, \mu)$. Evaluating the inner products with $\omega_{k}$ gives

$$
\begin{aligned}
& \left.X_{X}^{R}\right\lrcorner \omega_{k}=\delta\left\langle\mu-k g^{-1} g^{\prime}, X\right\rangle \\
& \left.X_{X}^{L}\right\lrcorner \omega_{k}=-\delta\left\langle g \mu g^{-1}, X\right\rangle,
\end{aligned}
$$

which shows that eqs. $(1.13 \mathrm{a}, \mathrm{b})$ give the moment maps generating these actions. To verify the Poisson bracket relations (1.15a-c), we compute:

$$
\begin{aligned}
\left\{J_{X}^{L}, J_{Y}^{L}\right\}=X_{X}^{L}\left(J_{Y}^{L}\right) & =\left\langle g \mu g^{-1},[X, Y]\right\rangle+k\left\langle X, Y^{\prime}\right\rangle \\
\left\{J_{X}^{R}, J_{Y}^{R}\right\}= & X_{X}^{R}\left(J_{Y}^{R}\right)=\left\langle-\mu+k g^{-1} g^{\prime},[X, Y]\right\rangle-k\left\langle X, Y^{\prime}\right\rangle \\
\left\{J_{X}^{L}, J_{Y}^{R}\right\} & =X_{X}^{L}\left(J_{Y}^{R}\right)=0 .
\end{aligned}
$$

Proposition 1.1 implies that the map

$$
\begin{aligned}
J_{k}^{L R}: T^{*} \widetilde{G} & \longrightarrow(\widetilde{\mathfrak{g}} \oplus \widetilde{\mathfrak{g}})^{*} \\
J_{k}^{L R}:(g, \mu) & \longmapsto\left(g \mu g^{-1},-\mu+k g^{-1} g^{\prime}\right)
\end{aligned}
$$

to the dual of the direct sum Lie algebra $\widetilde{\mathfrak{g}} \oplus \widetilde{\mathfrak{g}}$ is a nonequivariant moment map generating the product of the left $(L)$ and right $(R)$ translations, the nonequivariance given by $k c^{L R}$, where $c^{L R}$ is the 2-cocycle

$$
\begin{aligned}
c^{L R}:(\widetilde{\mathfrak{g}} \oplus \widetilde{\mathfrak{g}}) \times(\widetilde{\mathfrak{g}} \oplus \widetilde{\mathfrak{g}}) & \longrightarrow \mathbb{R} \\
c^{L R}\left(\left(X_{1}, Y_{1}\right),\left(X_{2}, Y_{2}\right)\right) & :=\left\langle X_{1}, X_{2}^{\prime}\right\rangle-\left\langle Y_{1}, Y_{2}^{\prime}\right\rangle .
\end{aligned}
$$


The centrally extended algebra $(\widetilde{\mathfrak{g}} \oplus \widetilde{\mathfrak{g}})^{\wedge}$ associated with this cocycle is identified as the space $\widetilde{\mathfrak{g}}+\widetilde{\mathfrak{g}}+\mathbb{R}$, with Lie bracket

$$
\left[\left(X_{1}, Y_{1}, a_{1}\right),\left(X_{2}, Y_{2}, a_{2}\right)\right]=\left(\left[X_{1}, X_{2}\right],\left[Y_{1}, Y_{2}\right],\left\langle X_{1}, X_{2}^{\prime}\right\rangle-\left\langle Y_{1}, Y_{2}^{\prime}\right\rangle\right)
$$

Again, $(\widetilde{\mathfrak{g}} \oplus \widetilde{\mathfrak{g}})^{\wedge}$ is identified as a dense subspace of the dual space $(\widetilde{\mathfrak{g}} \oplus \widetilde{\mathfrak{g}})^{\wedge *}$ through the pairing

$$
\begin{aligned}
& \langle(U, V, a),(X, Y, b)\rangle:=\langle U, X\rangle+\langle V, Y\rangle+a b, \\
& (U, V, a) \in(\widetilde{\mathfrak{g}} \oplus \widetilde{\mathfrak{g}})^{\wedge *},(X, Y, b) \in(\widetilde{\mathfrak{g}} \oplus \widetilde{\mathfrak{g}})^{\wedge} .
\end{aligned}
$$

The extended coadjoint representation on $(\widetilde{\mathfrak{g}} \oplus \widetilde{\mathfrak{g}})^{\wedge *}$ is given by the formulae

$$
\begin{aligned}
\widehat{a d}_{(X, Y, b)}^{*}(U, V, a) & =\left(a d_{X}^{*} U+a X^{\prime}, a d_{Y}^{*} V-a Y^{\prime}, 0\right) \\
\widehat{A d}_{(\widehat{g, h})}^{*}(U, V, a) & =\left(A d_{g}^{*} U+a g^{\prime} g^{-1}, A d_{h}^{*} V-a h^{\prime} h^{-1}, a\right),
\end{aligned}
$$

where again, the centrally extended group $(\widetilde{G} \times \widetilde{G})^{\wedge} \rightarrow \widetilde{G} \times \widetilde{G}$ acts through the projection of any element $(\widehat{g, h}) \in(\widetilde{G} \times \widetilde{G})^{\wedge}$ to its image $(g, h)$ in $\widetilde{G} \times \widetilde{G}$.

From Proposition 1.1 we then have :

Corollary 1.2. The map

$$
\begin{aligned}
& \hat{J}_{k}^{L R}: T^{*} \widetilde{G} \longrightarrow(\tilde{\mathfrak{g}} \oplus \widetilde{\mathfrak{g}})^{\wedge *} \\
& \hat{J}_{k}^{L R}:(g, \mu) \longmapsto\left(g \mu g^{-1},-\mu+k g^{-1} g^{\prime}, k\right)
\end{aligned}
$$

is an equivariant moment map with respect to the extended coadjoint action (1.22a,b), generating the product action $L \times R$ of $\widetilde{G} \times \widetilde{G}$ on $T^{*} \widetilde{G}$.

The Lie Poisson bracket on $(\widetilde{\mathfrak{g}} \oplus \widetilde{\mathfrak{g}})^{\wedge *}$ is determined by:

$$
\begin{aligned}
\left\{\left\langle\cdot \mid\left(X_{1}, Y_{1}, b_{1}\right)\right\rangle,\right. & \left.\left\langle\cdot \mid\left(X_{2}, Y_{2}, b_{2}\right)\right\rangle\right\}\left.\right|_{(U, V, a)} \\
& =\left\langle U,\left[X_{1}, X_{2}\right]\right\rangle+\left\langle V,\left[Y_{1}, Y_{2}\right]\right\rangle+a\left(\left\langle X_{1}, X_{2}^{\prime}\right\rangle-\left\langle Y_{1}, Y_{2}^{\prime}\right\rangle\right)
\end{aligned}
$$

where $\langle. \mid(X, Y, b)\rangle$ again denotes the linear functional on $(\widetilde{\mathfrak{g}} \oplus \widetilde{\mathfrak{g}})^{\wedge *}$ corresponding to an element $(X, Y, b) \in(\widetilde{\mathfrak{g}} \oplus \widetilde{\mathfrak{g}})^{\wedge}$. The moment map $\hat{J}_{k}^{L R}$ is thus a Poisson map with respect to the Poisson brackets (1.10a-c) associated to the noncanonical symplectic form $\omega_{k}$. 
1c. Hamiltonian Action of $\widetilde{G} \ltimes \widetilde{\mathfrak{g}}_{A}$ on $T^{*} \widetilde{G}$

Viewing $\widetilde{\mathfrak{g}}$ as an additive group, denoted $\widetilde{\mathfrak{g}}_{A}$, upon which $\widetilde{G}$ acts via the adjoint representation, we may form the semi-direct product $\widetilde{G} \ltimes \widetilde{\mathfrak{g}}_{A}$, with group multiplication defined by

$$
\begin{gathered}
\left(\widetilde{G} \ltimes \widetilde{\mathfrak{g}}_{A}\right) \times\left(\widetilde{G} \ltimes \widetilde{\mathfrak{g}}_{A}\right) \rightarrow \widetilde{G} \ltimes \widetilde{\mathfrak{g}}_{A} \\
(g, X) \times(h, Y):=\left(g h, X+g Y g^{-1}\right) \\
g, h \in \widetilde{G}, \quad X, Y \in \widetilde{\mathfrak{g}}_{A} .
\end{gathered}
$$

The corresponding Lie algebra is the semi-direct sum $\widetilde{\mathfrak{g}} \dot{+} \widetilde{\mathfrak{g}}_{A}$, identified as a vector space with $\widetilde{\mathfrak{g}}+\widetilde{\mathfrak{g}}$, with Lie bracket

$$
\left[\left(X_{1}, Y_{1}\right),\left(X_{2}, Y_{2}\right)\right]:=\left(\left[X_{1}, X_{2}\right],\left[X_{1}, Y_{2}\right]-\left[X_{2}, Y_{1}\right]\right)
$$

The dual space $\left(\widetilde{\mathfrak{g}}+\widetilde{\mathfrak{g}}_{A}\right)^{*}$ is again identified with $\widetilde{\mathfrak{g}}+\widetilde{\mathfrak{g}}_{A}$, with dual pairing

$$
\begin{aligned}
\langle(U, V),(X, Y)\rangle & :=\langle V, X\rangle+\langle U, Y\rangle \\
(U, V) & \in\left(\widetilde{\mathfrak{g}}+\widetilde{\mathfrak{g}}_{A}\right)^{*},(X, Y) \in \widetilde{\mathfrak{g}} \dot{+} \widetilde{\mathfrak{g}}_{A} .
\end{aligned}
$$

(Note the reversal of conventions relative to (1.21).) The coadjoint representation of elements $(X, Y) \in \widetilde{\mathfrak{g}} \dot{+} \widetilde{\mathfrak{g}}_{A},(g, Y) \in \widetilde{G} \ltimes \widetilde{\mathfrak{g}}_{A}$ in the algebra and group is given by

$$
\begin{aligned}
a d_{(X, Y)}^{*}(U, V) & =([X, U],[X, V]+[Y, U]) \\
A d_{(g, Y)}^{*}(U, V) & =\left(g U g^{-1}, g V g^{-1}+\left[Y, g U g^{-1}\right]\right) .
\end{aligned}
$$

The following action of the additive group $\widetilde{\mathfrak{g}}_{A}$ on $T^{*} \widetilde{G}$ is easily verified to leave the canonical symplectic form $\omega_{0}$ invariant

$$
\begin{gathered}
A_{X}:(g, \mu) \longmapsto\left(g, \mu+\left(g^{-1} X g\right)^{\prime}\right) \\
X \in \widetilde{\mathfrak{g}}_{A} .
\end{gathered}
$$

In the remainder of this subsection only the canonical symplectic structure on $T^{*} \widetilde{G}$ will be used. 
Lemma 1.3. The $\widetilde{\mathfrak{g}}_{A}$ - action (1.29) is Hamiltonian, and is generated by the equivariant moment map $J^{A}: T^{*} \widetilde{G} \longrightarrow \mathfrak{g}_{A}^{*}$ defined by the formula

$$
J^{A}=g^{\prime} g^{-1}
$$

(where the dual pairing (1.2) is applied to $\widetilde{\mathfrak{g}}_{A} \sim \widetilde{\mathfrak{g}}, \widetilde{\mathfrak{g}}_{A}^{*} \sim \widetilde{\mathfrak{g}}^{*}$.)

Proof. Differentiating (1.29) along a 1-parameter subgroup $\{-t X\}$ with respect to $t$ shows that the infinitesimal action is generated by the vector field

$$
X_{X}^{A}=-\left\langle\left(g^{-1} X g\right)^{\prime}, \frac{\delta}{\delta \mu}\right\rangle \text {. }
$$

Taking the inner product with $\omega_{0}$ gives

$$
\begin{aligned}
\left.X_{X}^{A}\right\lrcorner \omega_{0} & =-\left\langle g^{-1} X g,\left(g^{-1} \delta g\right)^{\prime}\right\rangle \\
& =-\delta\left\langle X, g^{\prime} g^{-1}\right\rangle,
\end{aligned}
$$

showing that the flow is generated by the Hamiltonian $-J_{X}^{A}$, where

$$
J_{X}^{A}:=\left\langle J^{A}, X\right\rangle .
$$

The equivariance follows from the fact that, for any $X, Y \in \widetilde{\mathfrak{g}}_{A}$, the functions $J_{X}^{A}, J_{Y}^{A}$ depend only on the first factor $g$ in $(g, \mu) \in T^{*} \widetilde{G}$, and hence, by (1.10a) they Poisson commute.

We may now compose this action with the left translation action (1.12) for $k=0$,

$$
L_{h}:(g, \mu) \longmapsto(h g, \mu)
$$

to obtain an action $L A:\left(\widetilde{G} \ltimes \mathfrak{g}_{A}\right) \times T^{*} \widetilde{G} \longrightarrow T^{*} \widetilde{G}$ of the semi-direct product group $\widetilde{G} \ltimes \widetilde{\mathfrak{g}}_{A}$ defined by:

$$
L A_{(h, X)}:(g, \mu) \longmapsto\left(h g, \mu+\left(g^{-1} h^{-1} X h g\right)^{\prime}\right) .
$$

It is easily verified that these maps compose correctly to define an action of $\widetilde{G} \ltimes \widetilde{\mathfrak{g}}_{A}$ on $T^{*} \widetilde{G}$ and, by Lemma 1.3 and the fact that the $L$-action (1.34) is Hamiltonian, the combined $L A$-action (1.35) is Hamiltonian as well. However, the resulting moment map

$$
\begin{aligned}
J^{L A}: T^{*} \widetilde{G} & \longrightarrow\left(\widetilde{\mathfrak{g}}+\widetilde{\mathfrak{g}}_{A}\right)^{*} \\
J^{L A}:(g, \mu) \longmapsto & \left(J^{A}(g, \mu), J^{L}(g, \mu)\right) \\
& =\left(g^{\prime} g^{-1}, g \mu g^{-1}\right)
\end{aligned}
$$

is no longer equivariant, as indicated in the following 
Proposition 1.4. The moment map $J^{L A}$ generating the $\widetilde{G} \ltimes \widetilde{\mathfrak{g}}_{A}$ action is nonequivariant, having as cocycle:

$$
\begin{aligned}
& c^{L A}:\left(\widetilde{\mathfrak{g}}+\widetilde{\mathfrak{g}}_{A}\right) \times\left(\widetilde{\mathfrak{g}}+\widetilde{\mathfrak{g}}_{A}\right) \longrightarrow \mathbb{R} \\
& c^{L A}\left(\left(X_{1}, Y_{1}\right),\left(X_{2}, Y_{2}\right)\right):=\left\langle X_{1}, Y_{2}^{\prime}\right\rangle-\left\langle X_{2}, Y_{1}^{\prime}\right\rangle .
\end{aligned}
$$

Thus, the Hamiltonian generators

$$
\begin{aligned}
& J_{(X, Y)}^{L A}:=\left\langle g \mu g^{-1}, X\right\rangle+\left\langle g^{\prime} g^{-1}, Y\right\rangle \\
& X \in \widetilde{\mathfrak{g}}, \quad Y \in \widetilde{\mathfrak{g}}_{A}
\end{aligned}
$$

satisfy the following Poisson bracket relations

$$
\left\{J_{\left(X_{1}, Y_{1}\right)}^{L A}, J_{\left(X_{2}, Y_{2}\right)}^{L A}\right\}=J_{\left(\left[X_{1}, X_{2}\right],\left[X_{1}, Y_{2}\right]-\left[X_{2}, Y_{1}\right]\right)}^{L A}+\left\langle X_{1}, Y_{2}^{\prime}\right\rangle-\left\langle X_{2}, Y_{1}^{\prime}\right\rangle
$$

Proof. Eq. (1.39) reduces to the following relations

$$
\begin{aligned}
& \left\{J_{X}^{L}, J_{Y}^{L}\right\}=J_{[X, Y]}^{L} \\
& \left\{J_{X}^{L}, J_{Y}^{A}\right\}=J_{[X, Y]}^{A}+\left\langle X, Y^{\prime}\right\rangle \\
& \left\{J_{X}^{A}, J_{Y}^{A}\right\}=0 .
\end{aligned}
$$

Eq. (1.40a) is the particular case of eq. (1.15a) with $k=0$, while eq. (1.40c) is implied by Lemma 1.3. Eq. (1.40b) is verified directly:

$$
\begin{aligned}
\left\{J_{X}^{L}, J_{Y}^{A}\right\} & =-X_{Y}^{A}\left(J_{X}^{L}\right) \\
& =\left\langle\left(g^{-1} Y g\right)^{\prime}, \frac{\delta}{\delta \mu}\right\rangle\left(\left\langle g \mu g^{-1}, X\right\rangle\right) \\
& =\left\langle g^{\prime} g^{-1},[X, Y]\right\rangle+\left\langle X, Y^{\prime}\right\rangle
\end{aligned}
$$

where eq. (1.31) was used in the second line.

The non-equivariance of the map $J^{L A}: T^{*} \widetilde{G} \rightarrow\left(\widetilde{\mathfrak{g}} \dot{+} \widetilde{\mathfrak{g}}_{A}\right)^{*}$ with respect to the group action (1.35) may be expressed in finite form as:

$$
J^{L A} \circ L A_{(h, X)}=A d_{(h, X)}^{*} \circ J^{L A}+C^{L A}(h, X),
$$


where the group 1-cocycle $C^{L A}: \widetilde{G} \ltimes \widetilde{\mathfrak{g}}_{A} \rightarrow\left(\widetilde{\mathfrak{g}} \dot{+} \mathfrak{g}_{A}\right)^{*}$ is defined by the formula:

$$
C^{L A}(h, X):=\left(h^{\prime} h^{-1}, X^{\prime}-\left[h^{\prime} h^{-1}, X\right]\right) .
$$

The cocycle relation

$$
C^{L A}\left(g h, X+g Y g^{-1}\right)=A d_{(g, X)}^{*} C^{L A}(h, Y)+C^{L A}(g, X)
$$

is verified by applying the $A d^{*}$-action (1.28b) to the definition (1.42).

Using the algebra 2 -cocycle $c^{L A}$ given in eq. (1.37), we define the centrally extended Lie algebra $\left(\widetilde{\mathfrak{g}} \dot{+} \widetilde{\mathfrak{g}}_{A}\right)^{\wedge}$ as the space $\widetilde{\mathfrak{g}}+\widetilde{\mathfrak{g}}+\mathbb{R}$, with Lie bracket

$$
\left[\left(X_{1}, Y_{1}, a_{1}\right),\left(X_{2}, Y_{2}, a_{2}\right)\right]=\left(\left[X_{1}, X_{2}\right],\left[X_{1}, Y_{2}\right]-\left[X_{2}, Y_{1}\right],\left\langle X_{1}, Y_{2}^{\prime}\right\rangle-\left\langle X_{2}, Y_{1}^{\prime}\right\rangle\right) \text {. }
$$

A dense subspace of the dual $\left(\widetilde{\mathfrak{g}} \dot{+} \widetilde{\mathfrak{g}}_{A}\right)^{\wedge *}$ is again identified with $\left(\widetilde{\mathfrak{g}}+\widetilde{\mathfrak{g}}_{A}\right)^{\wedge}$, with typical elements denoted again $(U, V, a)$. The notational conventions are such that the pairing of eq. (1.21) is replaced by:

$$
\begin{gathered}
\langle(U, V, a),(X, Y, b)\rangle_{A}:=\langle V, X\rangle+\langle U, Y\rangle+a b \\
(U, V, a) \in\left(\widetilde{\mathfrak{g}} \oplus \widetilde{\mathfrak{g}}_{A}\right)^{\wedge *}, \quad(X, Y, b) \in\left(\widetilde{\mathfrak{g}} \oplus \widetilde{\mathfrak{g}}_{A}\right)^{\wedge}
\end{gathered}
$$

The extended coadjoint representation on $\left(\widetilde{\mathfrak{g}} \dot{+} \widetilde{\mathfrak{g}}_{A}\right)^{\wedge *}$ is then given by the formulae:

$$
\begin{aligned}
\widehat{a d}_{(X, Y, a)}^{*}(U, V, b) & =\left([X, U]+b X^{\prime},[X, V]+[Y, U]+b Y^{\prime}, 0\right) \\
\widehat{A d}_{(\widehat{g, Y})}^{*}(U, V, b) & =\left(g U g^{-1}+b g^{\prime} g^{-1}, g V g^{-1}+\left[Y, g U g^{-1}\right]+b Y^{\prime}-b\left[g^{\prime} g^{-1}, Y\right], b\right) .
\end{aligned}
$$

From Proposition 1.4 then follows

Corollary 1.5. The map

$$
\begin{aligned}
& \hat{J}^{L A}: T^{*} \widetilde{G} \longrightarrow\left(\widetilde{\mathfrak{g} \oplus} \widetilde{\mathfrak{g}}_{A}\right)^{\wedge *} \\
& \hat{J}^{L A}:(g, \mu) \longmapsto\left(g^{\prime} g^{-1}, g \mu g^{-1}, 1\right)
\end{aligned}
$$

is an equivariant moment map with respect to the extended coadjoint action (1.45a,b), generating the action $L A:\left(\widetilde{G} \ltimes \widetilde{\mathfrak{g}}_{A}\right) \times T^{*} \widetilde{G} \rightarrow T^{*} \widetilde{G}$ defined by (1.35).

The Lie-Poisson bracket on $\left(\widetilde{\mathfrak{g}} \dot{+} \widetilde{\mathfrak{g}}_{A}\right)^{\wedge *}$ is determined by the formula:

$$
\begin{aligned}
& \left\{\left\langle\cdot \mid\left(X_{1}, Y_{1}, b_{1}\right)\right\rangle_{A},\left.\left\langle\cdot \mid\left(X_{2}, Y_{2}, b_{2}\right)\right\rangle_{A}\right|_{(U, V, a)}\right. \\
& \quad=\left\langle V,\left[X_{1}, X_{2}\right]\right\rangle+\left\langle U,\left[X_{1}, Y_{2}\right]-\left[X_{2}, Y_{1}\right]\right\rangle+a\left(\left\langle X_{1}, Y_{2}^{\prime}\right\rangle-\left\langle X_{2}, Y_{1}^{\prime}\right\rangle\right) .
\end{aligned}
$$


The moment map $\hat{J}^{L A}$ is thus a Poisson map with respect to the canonical Poisson brackets on $T^{*} \widetilde{G}$ given by setting $k=0$ in eqs. (1.10a-c).

1d. Hamiltonian Action of $\mathcal{D}_{1} \ltimes \mathcal{D}_{0}$

Let $\mathcal{D}_{1}:=$ Diff $S^{1}$ (resp. Diff $\mathbb{R}$ ) be the group of diffeomorphisms of $S^{1}$ (resp. $\mathbb{R}$ ) and $\mathcal{D}_{0}:=\mathcal{F}^{\times}\left(S^{1}\right)\left(\right.$ resp. $\left.\mathcal{F}^{\times}(\mathbb{R})\right)$ the space of smooth non-vanishing functions on $S^{1}$ (resp. $\mathbb{R})$, viewed as an abelian group under multiplication. Using the natural action of $\mathcal{D}_{1}$ on $\mathcal{D}_{0}$

$$
\begin{aligned}
\widetilde{\sigma}: \mathcal{D}_{0} & \rightarrow \mathcal{D}_{0} \\
\widetilde{\sigma}: f & \longmapsto f \circ \widetilde{\sigma}^{-1} \\
\widetilde{\sigma} & \in \mathcal{D}_{1},
\end{aligned}
$$

we define the semi-direct product $\mathcal{D}_{1} \ltimes \mathcal{D}_{0}$, with group multiplication

$$
\begin{aligned}
&\left(\mathcal{D}_{1} \ltimes \mathcal{D}_{0}\right) \times\left(\mathcal{D}_{1} \ltimes \mathcal{D}_{0}\right) \longrightarrow \mathcal{D}_{1} \ltimes \mathcal{D}_{0} \\
&\left(\widetilde{\sigma}, f_{1}\right) \times\left(\widetilde{\sigma}_{2}, \widetilde{f}_{2}\right) \longmapsto\left(\widetilde{\sigma}_{1} \circ \widetilde{\sigma}_{2}, f_{1} f_{2} \circ \widetilde{\sigma}_{1}^{-1}\right) \\
& \widetilde{\sigma}_{1}, \widetilde{\sigma}_{2} \in \mathcal{D}_{1}, \quad f_{1}, f_{2} \in \mathcal{D}_{0} .
\end{aligned}
$$

The natural action of $\mathcal{D}_{1} \ltimes \mathcal{D}_{0}$ on the space $\mathcal{F}$ of smooth functions on $S^{1}$ (resp. $\mathbb{R})$ is:

$$
\begin{aligned}
& (\widetilde{\sigma}, f): \mathcal{F} \longrightarrow \mathcal{F} \\
& (\widetilde{\sigma}, f): h \longmapsto f h \circ \widetilde{\sigma}^{-1} .
\end{aligned}
$$

The Lie algebra of $\mathcal{D}_{1} \ltimes \mathcal{D}_{0}$ may be identified with the space diff 1 of differential operators of order $\leq 1$, with typical elements $\alpha \frac{d}{d \sigma}+\beta \in \operatorname{diff}_{1}$ denoted by pairs $(\alpha, \beta) \in \mathcal{F} \oplus \mathcal{F}$, and the Lie product given by commutation:

$$
\left[\left(\alpha_{1}, \beta_{1}\right),\left(\alpha_{2}, \beta_{2}\right)\right]=\left(\alpha_{1} \alpha_{2}^{\prime}-\alpha_{2} \alpha_{1}^{\prime}, \alpha_{1} \beta_{2}^{\prime}-\alpha_{2} \beta_{1}^{\prime}\right) .
$$

The dual space $\operatorname{diff}_{1}{ }^{*}$ is identified with the space $\mathcal{F} \oplus \mathcal{F}$ through the dual pairing

$$
\begin{gathered}
\operatorname{diff}_{1}^{*} \times \operatorname{diff}_{1} \rightarrow \mathbb{R} \\
\langle(v, w),(\alpha, \beta)\rangle:=\langle v \alpha\rangle+\langle w, \beta\rangle \\
(v, w) \in \operatorname{diff}_{1}^{*}, \quad(\alpha, \beta) \in \operatorname{diff}_{1},
\end{gathered}
$$


where the integrals

$$
\langle v, \alpha\rangle:=\int v(\sigma) \alpha(\sigma) d \sigma, \quad\langle w, \beta\rangle:=\int w(\sigma) \beta(\sigma) d \sigma
$$

are taken over the appropriate domain $\left(S^{1}\right.$ or $\left.\mathbb{R}\right)$, and the functions $\alpha, \beta, u, v \in \mathcal{F}$ are assumed to be integrable as required.

Let

$$
\begin{gathered}
\Theta: \mathbb{R}^{\times} \times \mathbb{R}^{\times} \longrightarrow G \\
\Phi: \mathbb{R}^{\times} \times \mathbb{R}^{\times} \longrightarrow G \\
\Theta(a, b):=\Theta_{0}(a) \Theta_{1}(b), \quad \Phi(a, b):=\Phi_{0}(a) \Phi_{1}(b) \\
a, b \in \mathbb{R}^{\times}
\end{gathered}
$$

be a pair of homomorphisms into $G$ from the direct product group $\mathbb{R}^{\times} \times \mathbb{R}^{\times}$formed from two copies of the multiplicative group $\mathbb{R}^{\times}$of non-zero reals. The derivatives $\left.\Theta_{*}\right|_{(1,1)} \in \operatorname{Hom}\left(\mathbb{R}^{2}, \mathfrak{g}\right),\left.\Phi_{*}\right|_{(1,1)} \in \operatorname{Hom}\left(\mathbb{R}^{2}, \mathfrak{g}\right)$ at the identity element $(1,1)$ may be expressed

$$
\left.\Theta_{*}\right|_{(1,1)}(x, y)=x \theta_{0}+y \theta_{1},\left.\quad \Phi_{*}\right|_{(1,1)}(x, y)=x \phi_{0}+y \phi_{1},
$$

where the elements $\theta_{0}, \theta_{1}, \phi_{0}, \phi_{1} \in \mathfrak{g}$ satisfy

$$
\left[\theta_{0}, \theta_{1}\right]=0, \quad\left[\phi_{0}, \phi_{1}\right]=0
$$

We define a class of right $\mathcal{D}_{1} \ltimes \mathcal{D}_{0}$ actions on $T^{*} \widetilde{G}$, parametrized by $(k, \Theta, \Phi)$ :

$$
\begin{gathered}
(\widetilde{\sigma}, f): T^{*} \widetilde{G} \longrightarrow T^{*} \widetilde{G} \\
(\widetilde{\sigma}, f):(g, \mu) \longmapsto(\check{g}, \check{\mu})
\end{gathered}
$$

where

$$
\begin{aligned}
& \check{g}:=\Theta_{1}\left(\widetilde{\sigma}^{\prime}\right) \Theta_{0}(f \circ \tilde{\sigma})(g \circ \tilde{\sigma}) \Phi_{0}(f \circ \tilde{\sigma})^{-1} \Phi_{1}\left(\widetilde{\sigma}^{\prime}\right)^{-1} \\
& \check{\mu}:=\widetilde{\sigma}^{\prime} \Phi_{1}\left(\widetilde{\sigma}^{\prime}\right) \Phi_{0}(f \circ \tilde{\sigma})(\mu \circ \widetilde{\sigma}) \Phi_{0}(f \circ \widetilde{\sigma})^{-1} \Phi_{1}\left(\widetilde{\sigma}^{\prime}\right)^{-1} \\
& +k \widetilde{\sigma}^{\prime}\left(f^{\prime} \circ \tilde{\sigma}\right) \Phi_{1}\left(\tilde{\sigma}^{\prime}\right) \Phi_{0}(f \circ \tilde{\sigma})(g \circ \tilde{\sigma})^{-1} \theta_{0}(g \circ \tilde{\sigma}) \Phi_{0}(f \circ \tilde{\sigma})^{-1} \Phi_{1}\left(\tilde{\sigma}^{\prime}\right)^{-1} \\
& +k \frac{\widetilde{\sigma}^{\prime \prime}}{\widetilde{\sigma}^{\prime}} \Phi_{1}\left(\widetilde{\sigma}^{\prime}\right) \Phi_{0}(f \circ \widetilde{\sigma})(g \circ \widetilde{\sigma})^{-1} \theta_{1}(g \circ \tilde{\sigma}) \Phi_{0}(f \circ \tilde{\sigma})^{-1} \Phi_{1}\left(\widetilde{\sigma}^{\prime}\right)^{-1} .
\end{aligned}
$$


Although this action appears complicated at first sight, it is actually very simple when decomposed as a product of the $\mathcal{D}_{0}$-action:

$$
(1, f):(g, \mu) \longmapsto\left(\Theta_{0}(f) g \Phi_{0}(f)^{-1}, \Phi_{0}(f) \mu \Phi_{0}(f)^{-1}+k f^{\prime} \Phi_{0}(f) g^{-1} \theta_{0} g \Phi_{0}(f)^{-1}\right)
$$

and the $\mathcal{D}_{1}$-action:

$$
(\widetilde{\sigma}, 1):(g, \mu) \longrightarrow(\check{g}, \check{\mu})
$$

defined by

$$
\begin{aligned}
& \check{g}=\Theta_{1}\left(\widetilde{\sigma}^{\prime}\right)(g \circ \tilde{\sigma}) \Phi_{1}\left(\widetilde{\sigma}^{\prime}\right)^{-1} \\
& \check{\mu}=\widetilde{\sigma}^{\prime} \Phi_{1}\left(\widetilde{\sigma}^{\prime}\right)(\mu \circ \widetilde{\sigma}) \Phi_{1}\left(\widetilde{\sigma}^{\prime}\right)^{-1}+k \frac{\widetilde{\sigma}^{\prime \prime}}{\widetilde{\sigma}^{\prime}} \Phi_{1}\left(\widetilde{\sigma}^{\prime}\right)(g \circ \widetilde{\sigma})^{-1} \theta_{1}(g \circ \widetilde{\sigma}) \Phi_{1}\left(\widetilde{\sigma}^{\prime}\right)^{-1}
\end{aligned}
$$

The $\mathcal{D}_{0}$-action $(1.58)$ is just left translation (1.12) by the element $\Theta_{0}(f) \in \widetilde{G}$ and right translation $(1.11)$ by the element $\Phi_{0}(f) \in \widetilde{G}$. The $\mathcal{D}_{1}$-action $(1.59 \mathrm{a}-\mathrm{c})$ is the cotangent bundle lift of the natural right action

$$
\begin{aligned}
& \widetilde{\sigma}: \widetilde{G} \longrightarrow \widetilde{G} \\
& \widetilde{\sigma}: g \longmapsto g \circ \widetilde{\sigma}
\end{aligned}
$$

composed with a "twisting" by the left translation action (1.12) of $\Theta_{1}\left(\widetilde{\sigma}^{\prime}\right) \in \widetilde{G}$ and right translation $(1.11)$ by $\Phi_{1}\left(\widetilde{\sigma}^{\prime}\right) \in \widetilde{G}$. It is verified directly that the maps (1.57a-c) do indeed compose correctly to define a right $\mathcal{D}_{1} \ltimes \mathcal{D}_{0}$ action. Moreover, by considering the separate maps that are composed to form $(1.57 \mathrm{a}-\mathrm{c})$, it is easily verified that this action preserves the symplectic form $\omega_{k}$ on $T^{*} \widetilde{G}$. The infinitesimal action obtained from $(1.57 \mathrm{a}-\mathrm{c})$ by differentiating the flow given by the $1-$ parameter group $\{\exp [-t(\alpha, \beta)] \subset$ $\left.\mathcal{D}_{1} \ltimes \mathcal{D}_{0}\right\}$ at $t=0$ is represented by the functional vector fields:

$$
X_{(\alpha, \beta)}^{\mathcal{D}}=X_{\alpha}^{1}+X_{\beta}^{0}
$$

where

$$
X_{\beta}^{0}:=X_{\beta \theta_{0}}^{L}+X_{\beta \phi_{0}}^{R}
$$

generates the $\mathcal{D}_{0}$-action $(1.58)$, and

$$
X_{\alpha}^{1}:=-\left\langle\alpha g^{\prime}, \frac{\delta}{\delta g}\right\rangle-\left\langle(\alpha \mu)^{\prime}, \frac{\delta}{\delta \mu}\right\rangle+X_{\alpha^{\prime} \theta_{1}}^{L}+X_{\alpha^{\prime} \phi_{1}}^{R}
$$


generates the $\mathcal{D}_{1}$-action $(1.59 \mathrm{a}-\mathrm{c})$. (In the above, we have chosen signs so that the map $(\alpha, \beta) \rightarrow X_{(\alpha, \beta)}^{\mathcal{D}}$ defines a homomorphism to the Lie algebra of functional vector fields on $T^{*} \widetilde{G}$, rather than an anti-homomorphism. This means the flows generate the left action of $\mathcal{D}_{1} \ltimes \mathcal{D}_{0}$ defined by replacing $(\widetilde{\sigma}, f)$ in $(1.57 \mathrm{a}-\mathrm{c})$ by the inverse $\left.\left(\widetilde{\sigma}^{-1},(f \circ \widetilde{\sigma})^{-1}\right)\right)$. The Hamiltonian properties of this action are summarized in the following:

Proposition 1.6. The action (1.57a-c) is Hamiltonian, with the 1-parameter subgroups $\{\exp [-t(\alpha, \beta)]\}$ generated as the flow of the Hamiltonians:

$$
\begin{aligned}
J_{(\alpha, \beta)}^{\mathcal{D}}:= & \left\langle J^{L}, \alpha^{\prime} \theta_{1}\right\rangle+\left\langle J^{R}, \alpha^{\prime} \phi_{1}\right\rangle \\
& +\frac{1}{2 k}\left(\left\langle\alpha J^{L}, J^{L}\right\rangle-\left\langle\alpha J^{R}, J^{R}\right\rangle\right. \\
& +\left\langle J^{L}, \beta \theta_{0}\right\rangle+\left\langle J^{R}, \beta \phi_{0}\right\rangle .
\end{aligned}
$$

Thus, the map

$$
\begin{aligned}
J^{\mathcal{D}}: T^{*} \widetilde{G} & \longrightarrow \operatorname{diff}_{1}{ }^{*} \\
J^{\mathcal{D}}:(g, \mu) & \longmapsto\left(J^{0}, J^{1}\right)
\end{aligned}
$$

defined by

$$
\begin{aligned}
J^{0}= & \mathcal{B}\left(J^{L}, \theta_{0}\right)+\mathcal{B}\left(J^{R}, \phi_{0}\right) \\
J^{1}= & -\mathcal{B}\left(J^{L \prime}, \theta_{1}\right)-\mathcal{B}\left(J^{R \prime}, \phi_{1}\right) \\
& +\frac{1}{2 k}\left(\mathcal{B}\left(J^{L}, J^{L}\right)-\mathcal{B}\left(J^{R}, J^{R}\right)\right)
\end{aligned}
$$

is the moment map generating this action. It is nonequivariant, satisfying the Poisson bracket relations:

$$
\begin{aligned}
\left\{J_{\left(\alpha_{1}, \beta_{1}\right)}^{\mathcal{D}}, J_{\left(\alpha_{2}, \beta_{2}\right)}^{\mathcal{D}}\right\}= & J_{\left[\alpha_{1} \alpha_{2}^{\prime}-\alpha_{2} \alpha_{1}^{\prime}, \alpha_{1} \beta_{2}^{\prime}-\alpha_{2} \beta_{1}^{\prime}\right]}^{\mathcal{D}} \\
& +k\left[\mathcal{B}\left(\theta_{0}, \theta_{0}\right)-\mathcal{B}\left(\phi_{0}, \phi_{0}\right)\right]\left\langle\beta_{1}, \beta_{2}^{\prime}\right\rangle \\
& +k\left[\mathcal{B}\left(\theta_{1}, \theta_{0}\right)-\mathcal{B}\left(\phi_{1}, \phi_{0}\right)\right]\left[\left\langle\alpha_{1}^{\prime}, \beta_{2}^{\prime}\right\rangle-\left\langle\alpha_{2}^{\prime}, \beta_{1}^{\prime}\right\rangle\right] \\
& +k\left[\mathcal{B}\left(\theta_{1}, \theta_{1}\right)-\mathcal{B}\left(\phi_{1}, \phi_{1}\right)\right]\left\langle\alpha_{1}^{\prime}, \alpha_{2}^{\prime \prime}\right\rangle .
\end{aligned}
$$

Proof. Let

$$
\begin{aligned}
& J_{\alpha}^{1}:=\left\langle J^{1}, \alpha\right\rangle=J_{(\alpha, 0)}^{\mathcal{D}} \\
& J_{\beta}^{0}:=\left\langle J^{0}, \beta\right\rangle=J_{(0, \beta)}^{\mathcal{D}} .
\end{aligned}
$$


To show that $J^{0}$ and $J^{1}$ generate the $\mathcal{D}_{0}$ and $\mathcal{D}_{1}$ actions respectively, we must verify

$$
\begin{aligned}
& \left.X_{\alpha}^{1}\right\lrcorner \omega_{k}=-\delta J_{\alpha}^{1} \\
& \left.X_{\beta}^{0}\right\lrcorner \omega_{k}=-\delta J_{\beta}^{0} .
\end{aligned}
$$

$J_{\alpha}^{1}$ and $J_{\beta}^{0}$ may be expressed in terms of the left and right moment maps $J^{L}, J^{R}$ as:

$$
\begin{aligned}
& J_{\alpha}^{1}=J_{\alpha^{\prime} \theta_{1}}^{L}+J_{\alpha^{\prime} \phi_{1}}^{R}+\frac{1}{2 k}\left(\left\langle\alpha J^{L}, J^{L}\right\rangle-\left\langle\alpha J^{R}, J^{R}\right\rangle\right) \\
& J_{\beta}^{0}=J_{\beta \theta_{0}}^{L}+J_{\beta \phi_{0}}^{R} .
\end{aligned}
$$

Eq. (1.66b) is immediate from the form $(1.61 b)$ of $X_{\beta}^{0}$ and eqs. (1.17a,b). Similarly, using the form (1.61c) of $X_{\alpha}^{1}$, eq (1.66a) reduces to:

$$
\left.-\left[\left\langle\alpha g^{\prime}, \frac{\delta}{\delta g}\right\rangle+\left\langle(\alpha \mu)^{\prime}, \frac{\delta}{\delta \mu}\right\rangle\right]\right\lrcorner \omega_{k}=\frac{1}{2 k} \delta\left[\left\langle\alpha J^{L}, J^{L}\right\rangle-\left\langle\alpha J^{R}, J^{R}\right\rangle\right],
$$

which is verified directly. The Poisson brackets (1.64) are equivalent to the relations:

$$
\begin{aligned}
\left\{J_{\alpha_{1}}^{1}, J_{\alpha_{2}}^{1}\right\} & =J_{\left(\alpha_{1} \alpha_{2}^{\prime}-\alpha_{2} \alpha_{1}^{\prime}\right\rangle}+k\left[\mathcal{B}\left(\theta_{1}, \theta_{1}\right)-\mathcal{B}\left(\phi_{1}, \phi_{1}\right)\right]\left\langle\alpha_{1}^{\prime}, \alpha_{2}^{\prime}\right\rangle \\
\left\{J_{\alpha}^{1}, J_{\beta}^{0}\right\} & =J_{\alpha \beta^{\prime}}^{0}+k\left[\mathcal{B}\left(\theta_{1}, \theta_{0}\right)-\mathcal{B}\left(\phi_{1}, \phi_{0}\right)\right]\left\langle\alpha^{\prime}, \beta^{\prime}\right\rangle \\
\left\{J_{\beta_{1}}^{0}, J_{\beta_{2}}^{0}\right\} & =k\left[\mathcal{B}\left(\theta_{0}, \theta_{0}\right)-\mathcal{B}\left(\phi_{0}, \phi_{0}\right)\right]\left\langle\beta_{1}, \beta_{2}^{\prime}\right\rangle .
\end{aligned}
$$

This is verified using the decompositions $(1.63 \mathrm{~b}, \mathrm{c})$ and the Poisson bracket relations

$$
\begin{aligned}
\left\{J_{X}^{L},\left\langle\alpha J^{L}, J^{L}\right\rangle\right\} & =-2 k J_{\alpha X^{\prime}}^{L} \\
\left\{J_{X}^{R},\left\langle\alpha J^{R}, J^{R}\right\rangle\right\} & =2 k J_{\alpha X^{\prime}}^{R} \\
\left\{\left\langle\alpha_{1} J^{L}, J^{L}\right\rangle,\left\langle\alpha_{2} J^{L}, J^{L}\right\rangle\right\} & =2 k\left\langle\left(\alpha_{1} \alpha_{2}^{\prime}-\alpha_{2} \alpha_{1}^{\prime}\right) J^{L}, J^{L}\right\rangle \\
\left\{\left\langle\alpha_{1} J^{R}, J^{R}\right\rangle,\left\langle\alpha_{2} J^{R}, J^{R}\right\rangle\right\} & =-2 k\left\langle\left(\alpha_{1} \alpha_{2}^{\prime}-\alpha_{2} \alpha_{1}^{\prime}\right) J^{R}, J^{R}\right\rangle,
\end{aligned}
$$

which follow from $(1.15 \mathrm{a}, \mathrm{b})$ and the Leibnitz rule.

In the Poisson brackets (1.64) we may identify three 2-cocycles for the algebra $\operatorname{diff}_{1}$

$$
\begin{aligned}
c_{i}^{\mathcal{D}}: \operatorname{diff}_{1} \times \operatorname{diff}_{1} & \rightarrow \mathbb{R}, \quad i=1,2,3 \\
c_{1}^{\mathcal{D}}\left(\left(\alpha_{1}, \beta_{1}\right),\left(\alpha_{2}, \beta_{2}\right)\right) & :=\left\langle\beta_{1}, \beta_{2}^{\prime}\right\rangle \\
c_{2}^{\mathcal{D}}\left(\left(\alpha_{1}, \beta_{1}\right),\left(\alpha_{2}, \beta_{2}\right)\right) & :=\left\langle\alpha_{1}^{\prime}, \beta_{2}^{\prime}\right\rangle-\left\langle\alpha_{2}^{\prime}, \beta_{1}^{\prime}\right\rangle \\
c_{3}^{\mathcal{D}}\left(\left(\alpha_{1}, \beta_{1}\right),\left(\alpha_{2}, \beta_{2}\right)\right) & :=\left\langle\alpha_{1}^{\prime}, \alpha_{2}^{\prime \prime}\right\rangle .
\end{aligned}
$$


Any combination of these may be used to define central extensions of the algebra. In particular, define the cocycle:

$$
c^{\mathcal{D}}:=l_{1} c_{1}^{\mathcal{D}}+l_{2} c_{2}^{\mathcal{D}}+l_{3} c_{3}^{\mathcal{D}}
$$

where

$$
\begin{aligned}
& l_{1}:=\mathcal{B}\left(\theta_{0}, \theta_{0}\right)-\mathcal{B}\left(\phi_{0}, \phi_{0}\right) \\
& l_{2}:=\mathcal{B}\left(\theta_{1}, \theta_{0}\right)-\mathcal{B}\left(\phi_{1}, \phi_{0}\right) \\
& l_{3}:=\mathcal{B}\left(\theta_{1}, \theta_{1}\right)-\mathcal{B}\left(\phi_{1}, \phi_{1}\right) .
\end{aligned}
$$

The centrally extended algebra $\operatorname{diff}_{1}^{\wedge\left(l_{1} l_{2} l_{3}\right)}$ associated with this cocycle is identified with the space $\operatorname{diff}_{1} \oplus \mathbb{R}$, with Lie brackets

$$
\begin{aligned}
{\left[\left(\alpha_{1}, \beta_{1}, a_{1}\right),\left(\alpha_{2}, \beta_{2}, a_{2}\right)\right]=} & \left(\alpha_{1} \alpha_{2}^{\prime}-\alpha_{2} \alpha_{1}^{\prime}, \alpha_{1} \beta_{2}^{\prime}-\alpha_{2} \beta_{1}^{\prime},\right. \\
& \left.l_{1}\left\langle\beta_{1}, \beta_{2}^{\prime}\right\rangle+l_{2}\left(\left\langle\alpha_{1}^{\prime}, \beta_{2}^{\prime}\right\rangle-\left\langle\alpha_{2}^{\prime}, \beta_{1}^{\prime}\right\rangle\right)+l_{3}\left\langle\alpha_{1}^{\prime}, \alpha_{2}^{\prime \prime}\right\rangle\right) \\
\left(\alpha_{1}, \beta_{1}\right),\left(\alpha_{2}, \beta_{2}\right) \in & \operatorname{diff}_{1}, \quad a_{1}, a_{2} \in \mathbb{R} .
\end{aligned}
$$

A dense subspace of the dual space $\operatorname{diff}_{1}^{\wedge}{ }^{\wedge}\left(l_{1} l_{2} l_{3}\right) *$ is as usual identified with $\operatorname{diff}_{1} \oplus \mathbb{R}$, through the pairing:

$$
\langle(v, w, a),(\alpha, \beta, b)\rangle_{\mathcal{D}}:=\langle v, \alpha\rangle+\langle w, \beta\rangle+a b
$$

The extended coadjoint representation of $\operatorname{diff}_{1}^{\wedge\left(l_{1} l_{2} l_{3}\right)}$ on $\operatorname{diff}_{1}^{\wedge\left(l_{1} l_{2} l_{3}\right) *}$ is given by the formula

$\widehat{a d}_{(\alpha, \beta, b)}^{*}(v, w, a)=\left(2 \alpha^{\prime} v+\alpha v^{\prime}+w \beta^{\prime}-a l_{2} \beta^{\prime \prime}-a l_{3} \alpha^{\prime \prime \prime}, \alpha^{\prime} w+\alpha w^{\prime}+a l_{1} \beta^{\prime}+a l_{2} \alpha^{\prime \prime}, 0\right)$.

It follows from Proposition 1.6 that we may define an extended, equivariant moment map from $T^{*} \widetilde{G}$ to $\operatorname{diff}_{1}^{\wedge}{ }^{\left(l_{1} l_{2} l_{3}\right) *}$ that generates the same $\mathcal{D}_{1} \ltimes \mathcal{D}_{0}$ action $(1.57 \mathrm{a}-\mathrm{c})$.

Corollary 1.7. The map

$$
\begin{aligned}
& \hat{J}^{\mathcal{D}}: T^{*} \widetilde{G} \longrightarrow \operatorname{diff}_{1}^{\wedge\left(l_{1} l_{2} l_{3}\right) *} \sim \operatorname{diff}_{1}^{*} \oplus \mathbb{R} \\
& \hat{J}^{\mathcal{D}}:(g, \mu) \longmapsto\left(J^{\mathcal{D}}, k\right)
\end{aligned}
$$


is an equivariant moment map with respect to the extended coadjoint action (1.75), generating the $\mathcal{D}_{1} \ltimes \mathcal{D}_{0}$ action $(1.5 \% a-c)$.

The Lie Poisson bracket on $\operatorname{diff}_{1}^{\wedge\left(l_{1} l_{2} l_{3}\right) *}$ is determined by the formula:

$$
\begin{aligned}
\left\{\left\langle\cdot \mid\left(\alpha_{1}, \beta_{1}, b_{1}\right)\right\rangle_{\mathcal{D}},\langle\cdot|\right. & \left.\left.\left(\alpha_{2}, \beta_{2}, b_{2}\right)\right\rangle_{\mathcal{D}}\right\}\left.\right|_{(v, w, a)} \\
=\langle v & \left., \alpha_{1} \alpha_{2}^{\prime}-\alpha_{2} \alpha_{1}^{\prime}\right\rangle+\left\langle w, \alpha_{1} \beta_{2}^{\prime}-\alpha_{2} \beta_{1}^{\prime}\right\rangle+a l_{1}\left\langle\beta_{1}, \beta_{2}^{\prime}\right\rangle \\
& \quad+a l_{2}\left(\left\langle\alpha_{1}^{\prime}, \beta_{2}^{\prime}\right\rangle-\left\langle\alpha_{2}^{\prime}, \beta_{1}^{\prime}\right\rangle\right)+a l_{3}\left\langle\alpha_{1}^{\prime}, \alpha_{2}^{\prime \prime}\right\rangle,
\end{aligned}
$$

where, as usual, $\langle\cdot \mid(\alpha, \beta, b)\rangle_{\mathcal{D}}$ denotes the linear functional on $\operatorname{diff}_{1}^{\wedge\left(l_{1} l_{2} l_{3}\right) *}$ associated by dual pairing to the algebra element $(\alpha, \beta, b) \in \operatorname{diff}_{1}^{\wedge\left(l_{1} l_{2} l_{3}\right)}$. The map (1.76) is thus a Poisson map with respect to the Poisson brackets (1.10a-c) on $T^{*} G$ associated to the symplectic form $\omega_{k}$, and the Lie Poisson brackets $(1.77)$ on $\operatorname{diff}_{1}^{\wedge\left(l_{1} l_{2} l_{3}\right) *}$.

We mention at this point that for the applications to integrable systems to be discussed in section 2 , a particular case of the $\mathcal{D}_{1} \ltimes \mathcal{D}_{0}$ group action (1.57a-c) will be considered; namely, when the elements $\theta_{0}, \theta_{1}, \phi_{0}, \phi_{1} \in \mathfrak{g}$ are of the form

$$
\theta_{0}=-2 \theta_{1}=-2 \phi_{1}=: 2 \theta, \quad \phi_{0}=0
$$

Hence

$$
l_{1}=-2 l_{2}=4 \mathcal{B}(\theta, \theta)=: 4 l, \quad l_{3}=0,
$$

and the cocycle $c^{\mathcal{D}}$ takes the form

$$
c^{\mathcal{D}}\left(\left(\alpha_{1}, \beta_{1}\right),\left(\alpha_{2}, \beta_{2}\right)\right)=l\left(4\left\langle\beta_{1}, \beta_{2}^{\prime}\right\rangle-2\left\langle\alpha_{1}^{\prime}, \beta_{2}^{\prime}\right\rangle+2\left\langle\alpha_{2}^{\prime}, \beta_{1}^{\prime}\right\rangle\right) \text {. }
$$

The Poisson brackets (1.77) thus reduce to (cf. eq. (2.36)):

$$
\begin{gathered}
\left\{\left\langle\cdot \mid\left(\alpha_{1}, \beta_{1}, b_{1}\right)\right\rangle_{\mathcal{D}},\left.\left\langle\cdot \mid\left(\alpha_{2}, \beta_{2}, b_{2}\right)\right\rangle_{\mathcal{D}}\right|_{(v, w, a)}\right. \\
=\left\langle v, \alpha_{1} \alpha_{2}^{\prime}-\alpha_{2} \alpha_{1}^{\prime}\right\rangle+\left\langle w, \alpha_{1} \beta_{2}^{\prime}-\alpha_{2} \beta_{1}^{\prime}\right\rangle+4 a l\left\langle\beta_{1}, \beta_{2}^{\prime}\right\rangle-2 a l\left(\left\langle\alpha_{1}^{\prime}, \beta_{2}^{\prime}\right\rangle-\left\langle\alpha_{2}^{\prime}, \beta_{1}^{\prime}\right\rangle\right) .
\end{gathered}
$$

Note that for $k \neq 0$ the maps $J^{\mathcal{D}}: T^{*} \widetilde{G} \rightarrow \operatorname{diff}_{1}^{*}, \quad \hat{J}^{\mathcal{D}}: T^{*} \widetilde{G} \rightarrow \operatorname{diff}_{1}^{\wedge}\left(l_{1} l_{2} l_{3}\right) *$ defined by eqs. (1.63a-c), (1.76) factor through the map $J^{L R}: T^{*} \widetilde{G} \rightarrow(\widetilde{\mathfrak{g}} \oplus \widetilde{\mathfrak{g}})^{*}$ or $\hat{J}^{L R}: T^{*} \widetilde{G} \rightarrow(\widetilde{\mathfrak{g}} \oplus \widetilde{\mathfrak{g}})^{\wedge *}$, suggesting that the $\mathcal{D}_{1} \ltimes \mathcal{D}_{0}$ group action (1.57a-c) also induces a Hamiltonian action on $(\widetilde{\mathfrak{g}} \oplus \widetilde{\mathfrak{g}})^{\wedge *}$. This is in fact the case, and the action is easily computed. 
Proposition 1.8. The moment map $\hat{J}^{L R}: T^{*} \widetilde{G} \rightarrow(\widetilde{\mathfrak{g}} \oplus \widetilde{\mathfrak{g}})^{\wedge *}$ is equivariant with respect to the $\mathcal{D}_{1} \ltimes \mathcal{D}_{0}$ action $\left(1.5^{7}\right.$ a-c) on $T^{*} \widetilde{G}$ and the following action on $(\widetilde{\mathfrak{g}} \oplus \widetilde{\mathfrak{g}})^{\wedge *}$ :

$$
\begin{aligned}
& (\widetilde{\sigma}, f):(\mathfrak{g} \oplus \widetilde{\mathfrak{g}})^{\wedge *} \rightarrow(\widetilde{\mathfrak{g}} \oplus \widetilde{\mathfrak{g}})^{\wedge *} \\
& (\widetilde{\sigma}, f):(U, V, a) \rightarrow(\check{U}, \check{V}, a)
\end{aligned}
$$

where

$$
\begin{aligned}
& \check{U}=\widetilde{\sigma}^{\prime} \Theta_{1}\left(\widetilde{\sigma}^{\prime}\right) \Theta_{0}(f \circ \widetilde{\sigma})(U \circ \widetilde{\sigma}) \Theta_{0}(f \circ \widetilde{\sigma})^{-1} \Theta_{1}\left(\widetilde{\sigma}^{\prime}\right)^{-1}+a(f \circ \widetilde{\sigma})^{\prime} \theta_{0}+a \frac{\tilde{\sigma}^{\prime \prime}}{\widetilde{\sigma}^{\prime}} \theta_{1} \\
& \check{V}=\widetilde{\sigma}^{\prime} \Phi_{1}\left(\widetilde{\sigma}^{\prime}\right) \Phi_{0}(f \circ \tilde{\sigma})(V \circ \widetilde{\sigma}) \Phi_{0}(f \circ \widetilde{\sigma})^{-1} \Phi_{1}\left(\widetilde{\sigma}^{\prime}\right)^{-1}-a(f \circ \widetilde{\sigma})^{\prime} \phi_{0}-a \frac{\widetilde{\sigma}^{\prime \prime}}{\widetilde{\sigma}^{\prime}} \phi_{1} .
\end{aligned}
$$

Proof. This is verified by directly substituting the RHS of eqs. $(1.57 \mathrm{~b}, \mathrm{c})$ into the definitions $(1.13 \mathrm{a}, \mathrm{b})$ of $J^{L}$ and $J^{R}$.

The Hamiltonian properties of this action are summarized in the following.

Theorem 1.9. For $k \neq 0$, the moment map $\hat{J}^{\mathcal{D}}$ factors into:

$$
\hat{J}^{\mathcal{D}}=\hat{J}^{d} \circ \hat{J}^{L R}
$$

where the map $\hat{J}^{d}:(\widetilde{\mathfrak{g}} \oplus \widetilde{\mathfrak{g}})^{\wedge *} \longrightarrow \operatorname{diff}_{1}^{\wedge\left(l_{1} l_{2} l_{3}\right) *}$, defined by the formulae

$$
\begin{aligned}
\hat{J}^{d}(U, V, a) & =\left(J^{d 1}, J^{d 0}, a\right) \\
J^{d 1}(U, V, a) & :=-\mathcal{B}\left(U^{\prime}, \theta_{1}\right)-\mathcal{B}\left(V^{\prime}, \phi_{1}\right)+\frac{1}{2 a}(\mathcal{B}(U, U)-\mathcal{B}(V, V)) \\
J^{d 0}(U, V, a) & :=\mathcal{B}\left(U, \theta_{0}\right)+\mathcal{B}\left(V, \phi_{0}\right)
\end{aligned}
$$

(for $a \neq 0$ ) is a Poisson map with respect to the Lie Poisson brackets (1.24) and (1.77) on $(\widetilde{\mathfrak{g}} \oplus \widetilde{\mathfrak{g}})^{\wedge *}$ and $\operatorname{diff}_{1}^{\wedge\left(l_{1} l_{2} l_{3}\right) *}$. The Hamiltonian flow generated by

$$
\hat{J}_{(\alpha, \beta, a)}^{d}:=\left\langle\hat{J}^{d},(\alpha, \beta, a)\right\rangle_{\mathcal{D}}
$$

generates the action (1.80a-c) for the 1-parameter subgroup $\{\exp [-t(\alpha, \beta, a)]\}$. Thus, we have the following commuting diagram of equivariant moment maps:

$$
\begin{aligned}
& T^{*} \widetilde{G} \stackrel{\hat{J}^{\mathcal{D}}}{\longrightarrow} \operatorname{diff}_{1}^{\wedge\left(l_{1} l_{2} l_{3}\right) *} \\
& \hat{J}^{L R} \searrow \quad \nearrow \hat{J}^{d} \\
& (\widetilde{\mathfrak{g}} \oplus \widetilde{\mathfrak{g}})^{\wedge *}
\end{aligned}
$$


Proof. The factorization property (1.81) is seen directly from eqs. $(1.63 \mathrm{~b}, \mathrm{c})$. The fact that the map $\hat{J}^{d}$ is Poisson with respect to the Lie Poisson structures on $(\widetilde{\mathfrak{g}} \oplus \widetilde{\mathfrak{g}})^{\wedge\left(l_{1} l_{2} l_{3}\right) *}$ and $\operatorname{diff}_{1} \wedge^{*}$ is proved by the identical computation used in proving Proposition 1.6. The vector fields generating the 1 -parameter subgroups acting through (1.80a-c) are easily computed to be:

$$
\begin{aligned}
X_{\alpha}^{d 1}= & -\left\langle\alpha^{\prime} U+\alpha^{\prime}\left[\theta_{1}, U\right]+\alpha U^{\prime}+a \alpha^{\prime \prime} \theta_{1}, \frac{\delta}{\delta U}\right\rangle \\
& -\left\langle\alpha^{\prime} V+\alpha^{\prime}\left[\phi_{1}, V\right]+\alpha V^{\prime}-a \alpha^{\prime \prime} \phi_{1}, \frac{\delta}{\delta V}\right\rangle
\end{aligned}
$$

for the $\mathcal{D}_{1}$-action, and

$$
X_{\beta}^{d 0}=-\left\langle\beta\left[\theta_{0}, U\right]+a \beta^{\prime} \theta_{0}, \frac{\delta}{\delta U}\right\rangle-\left\langle\beta\left[\phi_{0}, V\right]-a \beta^{\prime} \phi_{0}, \frac{\delta}{\delta V}\right\rangle
$$

for the $\mathcal{D}_{0}$-action. Using the definition (1.24) of the Lie Poisson bracket on $(\widetilde{\mathfrak{g}} \oplus \widetilde{\mathfrak{g}})^{\wedge *}$, together with the Leibnitz rule, we find:

$$
\begin{aligned}
& X_{\alpha}^{d 1}(\langle\cdot \mid(X, Y, b)\rangle)=\left\{\hat{J}_{(\alpha, 0,0)}^{d},\langle\cdot \mid(X, Y, b)\rangle\right\} \\
& X_{\beta}^{d 0}(\langle\cdot \mid(X, Y, b)\rangle)=\left\{\hat{J}_{(0, \beta, 0)}^{d},\langle\cdot \mid(X, Y, b)\rangle\right\},
\end{aligned}
$$

and hence $\hat{J}^{d}$ is the moment map generating the action (1.80a-c) through Hamiltonian flows.

Note that the moment map $J^{\mathcal{D}}=\left(J^{0}, J^{1}\right)$ defined in eqs. (1.63a-c) may be expressed in a form that is equally valid whether $k$ vanishes or not:

$$
\begin{aligned}
J^{0}= & \mathcal{B}\left(g \mu g^{-1}, \theta_{0}\right)+\mathcal{B}\left(-\mu+k g^{-1} g^{\prime}, \phi_{0}\right) \\
J^{1}= & -\mathcal{B}\left(\left(g \mu g^{-1}\right)^{\prime}, \theta_{1}\right)-\mathcal{B}\left(-\mu^{\prime}+k\left(g^{-1} g^{\prime}\right)^{\prime}, \phi_{1}\right) \\
& +\mathcal{B}\left(g \mu g^{-1}, g^{\prime} g^{-1}\right)+\frac{k}{2} \mathcal{B}\left(g^{\prime} g^{-1}, g^{\prime} g^{-1}\right) .
\end{aligned}
$$

However, for $k=0$, these maps do not factor through $\hat{J}^{L R}: T^{*} \widetilde{G} \rightarrow(\widetilde{\mathfrak{g}} \oplus \widetilde{\mathfrak{g}})^{\wedge *}$. In the next subsection, two twisted $\mathcal{D}_{1} \ltimes \mathcal{D}_{0}$ actions that are Hamiltonian on $T^{*} \widetilde{G}$ will be considered, whose moment maps, while not factoring through $\hat{J}^{L R}$, do instead factor through the moment map $\hat{J}^{L A}: T^{*} \widetilde{G} \rightarrow\left(\widetilde{\mathfrak{g}} \oplus \widetilde{\mathfrak{g}}_{A}\right)^{\wedge *}$ of Corollary 1.5. The first is just a 
special case of the above, with the homomorphism $\Phi: \mathbb{R}^{\times} \times \mathbb{R}^{\times} \rightarrow G$ chosen as trivial, while the second is a new form of "twisted" $\mathcal{D}_{1} \ltimes \mathcal{D}_{0}$-action.

1e. Canonical Symplectic Structure: Factorization Through $\hat{J}^{L A}: T^{*} \widetilde{G} \rightarrow\left(\widetilde{\mathfrak{g}} \dot{+} \widetilde{\mathfrak{g}}_{A}\right)^{\wedge *}$

First, consider the $k=0$ case of the $\mathcal{D}_{1} \ltimes \mathcal{D}_{0}$ action (1.57a-c), with $\Phi$ trivial; i.e. $\phi_{0}=\phi_{1}=0$. From eq. $(1.85 \mathrm{a}-\mathrm{b})$, we see that the moment map $J^{\mathcal{D}}=\left(J^{0}, J^{1}\right)$ may be expressed as

$$
\begin{aligned}
J^{0} & =\mathcal{B}\left(J^{L}, \theta_{0}\right) \\
J^{1} & =-\mathcal{B}\left(J^{L^{\prime}}, \theta_{1}\right)+\mathcal{B}\left(J^{L}, J^{A}\right),
\end{aligned}
$$

and thus factors through $\hat{J}^{L A}: T^{*} \widetilde{G} \rightarrow\left(\widetilde{\mathfrak{g}} \oplus \widetilde{\mathfrak{g}}_{A}\right)^{\wedge *}$. The corresponding induced action on $\left(\widetilde{\mathfrak{g}} \oplus \widetilde{\mathfrak{g}}_{A}\right)^{\wedge *}$ is easily computed to be:

$$
(\widetilde{\sigma}, f):(U, V, a) \rightarrow(\check{U}, \check{V}, a)
$$

where

$$
\begin{aligned}
\check{U}:= & \widetilde{\sigma}^{\prime} \Theta_{1}\left(\widetilde{\sigma}^{\prime}\right) \Theta_{0}(f \circ \widetilde{\sigma})(U \circ \tilde{\sigma}) \Theta_{0}(f \circ \tilde{\sigma})^{-1} \Theta_{1}\left(\widetilde{\sigma}^{\prime}\right)^{-1} \\
\check{V}:= & \widetilde{\sigma}^{\prime} \Theta_{1}\left(\widetilde{\sigma}^{\prime}\right) \Theta_{0}(f \circ \widetilde{\sigma})(V \circ \widetilde{\sigma}) \Theta_{0}(f \circ \widetilde{\sigma})^{-1} \Theta_{1}\left(\widetilde{\sigma}^{\prime}\right)^{-1} \\
& +\widetilde{\sigma}^{\prime}\left(\frac{f^{\prime}}{f} \circ \widetilde{\sigma}\right) \theta_{0}+\frac{\widetilde{\sigma}^{\prime \prime}}{\widetilde{\sigma}^{\prime}} \theta_{1} .
\end{aligned}
$$

The vector field generating the action of the 1-parameter subgroup $\{\exp [-t(\alpha, \beta)]\}$ is:

$$
X_{(\alpha, \beta)}^{d_{a}}=X_{\alpha}^{d 0_{a}}+X_{\beta}^{d 1_{a}}
$$

where

$$
\begin{aligned}
& X_{\beta}^{d 0_{a}}:=-\left\langle\beta\left[\theta_{0}, V\right], \frac{\delta}{\delta V}\right\rangle-\left\langle\beta\left[\theta_{0}, U\right]+\beta^{\prime} \theta_{0}, \frac{\delta}{\delta U}\right\rangle \\
& X_{\alpha}^{d 1_{a}}:=-\left\langle(\alpha V)^{\prime}+\alpha^{\prime}\left[\theta_{1}, V\right], \frac{\delta}{\delta U}\right\rangle-\left\langle(\alpha U)^{\prime}+\alpha^{\prime}\left[\theta_{1}, U\right]+\alpha^{\prime \prime} \theta_{1}, \frac{\delta}{\delta U}\right\rangle .
\end{aligned}
$$

The action $(1.89 \mathrm{a}-\mathrm{c})$ is again Hamiltonian, and generated by the moment map

$$
\begin{aligned}
J^{d_{a}}:\left(\widetilde{\mathfrak{g}}+\widetilde{\mathfrak{g}}_{A}\right)^{\wedge *} & \longrightarrow \operatorname{diff}_{1}^{*} \\
J^{d_{a}}:(U, V, a) & \longrightarrow\left(J^{d 0_{a}}, J^{d 1_{a}}\right),
\end{aligned}
$$


where

$$
\begin{aligned}
J^{d 0_{a}} & :=\frac{1}{a} \mathcal{B}\left(V, \theta_{0}\right) \\
J^{d 1_{a}} & :=-\frac{1}{a} \mathcal{B}\left(V^{\prime}, \theta_{1}\right)+\frac{1}{a} \mathcal{B}(U, V) .
\end{aligned}
$$

In this case, both the maps $J^{\mathcal{D}}: T^{*} \widetilde{G} \rightarrow \operatorname{diff}_{1}^{*}$ and $J^{d_{a}}:\left(\mathfrak{g}+\widetilde{\mathfrak{g}}_{A}\right)^{\wedge *} \rightarrow \operatorname{diff}_{1}^{*}$ are equivariant, satisfying:

$$
\begin{aligned}
& \left\{J_{\left(\alpha_{1}, \beta_{1}\right)}^{\mathcal{D}}, J_{\left(\alpha_{2}, \beta_{2}\right)}^{\mathcal{D}}\right\}=J_{\left(\alpha_{1} \alpha_{2}^{\prime}-\alpha_{2} \alpha_{1}^{\prime}, \alpha_{1} \beta_{2}^{\prime}-\alpha_{2} \beta_{1}^{\prime}\right)}^{\mathcal{D}} \\
& \left\{J_{\left(\alpha_{1}, \beta_{1}\right)}^{d_{a}}, J_{\left(\alpha_{2}, \beta_{2}\right)}^{d_{a} \alpha_{2}}\right\}=J_{\left(\alpha_{1} \alpha_{2}^{\prime}-\alpha_{2} \alpha_{1}^{\prime}, \alpha_{1} \beta_{2}^{\prime}-\alpha_{2} \beta_{1}^{\prime}\right)}^{d_{a}}
\end{aligned}
$$

These are therefore Poisson maps with respect to the Lie Poisson structure on $\operatorname{diff}_{1}{ }^{*}$ without central extension. Summarizing, we have:

Proposition 1.10. For $k=0$, the moment map $J^{\mathcal{D}}$ factors into:

$$
J^{\mathcal{D}}=J^{d_{a}} \circ \hat{J}^{L A}
$$

We thus have the commuting diagram of equivariant moment maps:

$$
\begin{aligned}
& T^{*} \widetilde{G} \stackrel{J^{\mathcal{D}}}{\longrightarrow} \operatorname{diff}_{1}^{*} \\
& \hat{J}^{L A} \searrow \quad \quad \quad J^{d_{a}} \\
& \left(\widetilde{\mathfrak{g}} \dot{+} \widetilde{\mathfrak{g}}_{A}\right)^{\wedge *}
\end{aligned}
$$

where $J^{\mathcal{D}}$ generates the $\mathcal{D}_{1} \ltimes \mathcal{D}_{0}$ action $(1.57 a-c)$, with $k=0, \phi_{0}=\phi_{1}=0$, and $J^{d_{A}}$ generates the action $(1.89 a-c)$.

Another "twisted" action of $\mathcal{D}_{1} \ltimes \mathcal{D}_{0}$, or rather of the subgroup $\mathcal{D}_{1} \ltimes \mathcal{D}_{0}^{+} \subset \mathcal{D}_{1} \ltimes \mathcal{D}_{0}$ consisting of $\{(\widetilde{\sigma}, f), f>0\}$, may be defined on $T^{*} \widetilde{G}$, one which is nonequivariant, but still factors through $\hat{J}^{L A}: T^{*} \widetilde{G} \rightarrow\left(\mathfrak{g} \dot{+} \widetilde{\mathfrak{g}}_{A}\right)^{\wedge *}$. Let $\Theta=\left(\Theta_{0}, \Theta_{1}\right): \mathbb{R}^{\times} \times \mathbb{R}^{\times} \rightarrow G$ again be a homomorphism, with derivative at the identity $(1,1)$ identified, as in eq. (1.55), with a pair $\theta_{0}, \theta_{1} \in \mathfrak{g}$ of commuting elements of $\mathfrak{g}$. Define a right action $\mathcal{D}_{1} \ltimes \mathcal{D}_{0}^{+}$: $T^{*} \widetilde{G} \rightarrow T^{*} \widetilde{G}$ by:

$$
(\widetilde{\sigma}, f):(g, \mu) \longrightarrow\left(\Theta_{1}\left(\widetilde{\sigma}^{\prime}\right)(g \circ \tilde{\sigma}), \tilde{\sigma}^{\prime}(\mu \circ \tilde{\sigma})+\left[\ln (f \circ \tilde{\sigma})(g \circ \tilde{\sigma})^{-1} \Theta_{0}(g \circ \widetilde{\sigma})\right]^{\prime}\right)
$$


It is easily verified that this does compose correctly to define a right action of $\mathcal{D}_{1} \ltimes \mathcal{D}_{0}^{+}$, and that this action leaves invariant the canonical symplectic form $\omega_{0}$ on $T^{*} \widetilde{G}$. The infinitesimal action obtained by differentiating the flow of $\{\exp [-t(\alpha, \beta)]\}$ given by (1.95) is represented by the vector field

$$
X_{(\alpha, \beta)}^{\mathcal{D}_{A}}:=X_{\alpha}^{1_{A}}+X_{\beta}^{0_{A}}
$$

where

$$
X_{\beta}^{0_{A}}:=X_{\beta \theta_{0}}^{A}
$$

generates the $\mathcal{D}_{0}^{+}$action, and

$$
X_{\alpha}^{1_{A}}:=-\left\langle\alpha g^{\prime}, \frac{\delta}{\delta g}\right\rangle-\left\langle(\alpha \mu)^{\prime}, \frac{\delta}{\delta \mu}\right\rangle+X_{\alpha^{\prime} \theta_{1}}^{L}
$$

generates the $\mathcal{D}_{1}$-action. Thus

$$
\begin{gathered}
X^{\mathcal{D}_{A}}: \operatorname{diff}_{1} \longrightarrow \chi\left(T^{*} \widetilde{G}\right) \\
X^{\mathcal{D}_{A}}:(\alpha, \beta) \longmapsto X_{(\alpha, \beta)}^{\mathcal{D}_{A}}
\end{gathered}
$$

defines a homomorphism to the Lie algebra $\chi\left(T^{*} \widetilde{G}\right)$ of functional derivations (vector fields) on $T^{*} \widetilde{G}$. The Hamiltonian properties of this action are given in the following

Proposition 1.11. For $k=0$, the $\mathcal{D}_{1} \ltimes \mathcal{D}_{0}^{+}$-action (1.95) is Hamiltonian, and generated by the moment map

$$
\begin{aligned}
J^{\mathcal{D}_{A}}: T^{*} \widetilde{G} & \longrightarrow \operatorname{diff}_{1}{ }^{*} \\
J^{\mathcal{D}_{A}}:(g, \mu) & \longmapsto\left(J^{1_{A}}, J^{0_{A}}\right),
\end{aligned}
$$

where

$$
\begin{aligned}
J^{0_{A}} & =\mathcal{B}\left(J^{A}, \theta_{0}\right) \\
J^{1_{A}} & =-\mathcal{B}\left(J^{L^{\prime}}, \theta_{1}\right)+\mathcal{B}\left(J^{L}, J^{A}\right) .
\end{aligned}
$$

This map is nonequivariant, satisfying the Poisson bracket relations:

$$
\left\{J_{\left(\alpha_{1}, \beta_{1}\right)}^{\mathcal{D}_{A}}, J_{\left(\alpha_{2}, \beta_{2}\right)}^{\mathcal{D}_{A}}\right\}=J_{\left(\alpha_{1} \alpha_{2}^{\prime}-\alpha_{2} \alpha_{1}^{\prime}, \alpha_{1} \beta_{2}^{\prime}-\alpha_{2} \beta_{1}^{\prime}\right)}^{\mathcal{D}_{A}}+\mathcal{B}\left(\theta_{0}, \theta_{1}\right)\left[\left\langle\alpha_{1}^{\prime}, \beta_{2}^{\prime}\right\rangle-\left\langle\alpha_{2}^{\prime}, \beta_{1}^{\prime}\right\rangle\right]
$$


where

$$
J_{(\alpha, \beta)}^{\mathcal{D}_{A}}:=\left\langle J^{\mathcal{D}_{A}},(\alpha, \beta)\right\rangle=\left\langle J^{1_{A}}, \alpha\right\rangle+\left\langle J^{0_{A}}, \beta\right\rangle .
$$

Proof. The equalities

$$
\begin{aligned}
& \left.X_{\beta}^{0_{A}}\right\lrcorner \omega_{0}=-\delta\left(J_{\beta \theta_{0}}^{A}\right) \\
& \left.X_{\alpha}^{1_{A}}\right\lrcorner \omega_{0}=-\delta\left(J_{\alpha^{\prime} \theta_{1}}^{L}\right)-\delta\left\langle\alpha J^{L}, J^{A}\right\rangle,
\end{aligned}
$$

which imply the formulae $(1.98 \mathrm{~b}, \mathrm{c})$ for the moment map $J^{\mathcal{D}_{A}}$ are directly verified from the definitions $(1.96 \mathrm{a}, \mathrm{b}),(1.8),(1.13 \mathrm{a})$ and (1.30) of $X^{0_{A}}, X^{1_{A}}, \omega_{0}, J^{L}$ and $J^{A}$, respectively. The Poisson brackets (1.99) are equivalent to the relations:

$$
\begin{aligned}
\left\{J_{\alpha_{1}}^{1_{A}}, J_{\alpha_{2}}^{1_{A}}\right\} & =X_{\alpha_{1}}^{1_{A}}\left(J_{\alpha_{2}}^{1_{A}}\right) \\
& =\left\{J_{\alpha_{1}^{\prime} \theta_{1}}^{L}+\left\langle\alpha_{1} J^{L}, J^{A}\right\rangle, J_{\alpha_{2}^{\prime} \theta_{1}}+\left\langle\alpha_{2} J^{L}, J^{A}\right\rangle\right\} \\
& =J_{\alpha_{1} \alpha_{2}^{\prime}-\alpha_{2} \alpha_{1}^{\prime}}^{1_{A}} \\
\left\{J_{\alpha}^{1_{A}}, J_{\beta}^{0_{A}}\right\} & =-X_{\beta}^{0_{A}}\left(J_{\alpha}^{1_{A}}\right) \\
& =\left\{J_{\alpha^{\prime} \theta_{1}}^{L}+\left\langle\alpha J^{L}, J^{A}\right\rangle, J_{\beta \theta_{0}}^{A}\right\} \\
& =J_{\alpha \beta^{\prime} \theta_{0}}^{A}+\mathcal{B}\left(\theta_{0}, \theta_{1}\right)\left\langle\alpha^{\prime}, \beta^{\prime}\right\rangle \\
\left\{J_{\beta_{1}}^{0_{A}}, J_{\beta_{2}}^{0_{A}}\right\} & =X_{\beta_{1}}^{0_{A}}\left(J_{\beta_{2}}^{0_{A}}\right) \\
& =\left\{J_{\beta_{1} \theta_{0}}^{A}, J_{\beta_{2} \theta_{0}}^{A}\right\} \\
& =0
\end{aligned}
$$

where

$$
J_{\alpha}^{1_{A}}:=\left\langle J^{1_{A}}, \alpha\right\rangle, \quad J_{\beta}^{0_{A}}:=\left\langle J^{0_{A}}, \beta\right\rangle .
$$

These may be verified either directly from the definitions (1.96b,c), (1.98a,b) of $X_{\beta}^{0_{A}}$, $X_{\alpha}^{1_{A}}, J^{0_{A}}$ and $J^{1_{A}}$, or with the help of the Poisson bracket relations:

$$
\begin{aligned}
\left\{J_{\alpha_{1}^{\prime} \theta_{1}}^{L},\left\langle\alpha_{2} J^{L}, J^{A}\right\rangle\right\} & =-J_{\alpha_{1}^{\prime \prime} \alpha_{2} \theta_{1}}^{L} \\
\left\{\left\langle\alpha_{1} J^{L}, J^{A}\right\rangle,\left\langle\alpha_{2} J^{L}, J^{A}\right\rangle\right\} & =\left\langle\left(\alpha_{1} \alpha_{2}^{\prime}-\alpha_{2} \alpha_{1}^{\prime}\right) J^{L}, J^{A}\right\rangle,
\end{aligned}
$$

which follow from eqs. (1.40a-c), together with the Leibnitz rule. 
Since the cocycle $c_{2}^{\mathcal{D}}$ (eq. (1.70b)) enters in (1.99) we proceed, as usual, defining the central extension $\operatorname{diff}_{1}^{\wedge(0,-l, 0)}$ as in eq. (1.73), with

$$
l_{1}=l_{3}=0, \quad l_{2}=\mathcal{B}\left(\theta_{1}, \theta_{0}\right)=:-l,
$$

which gives the extended coadjoint representation

$$
\hat{a} d_{(\alpha, \beta, b)}^{*}(v, w, a)=\left(v^{\prime} \alpha+2 v \alpha^{\prime}+w \beta^{\prime}+a l \beta^{\prime \prime},(w \alpha)^{\prime}-a l \alpha^{\prime \prime}, 0\right)
$$

We then have

Corollary 1.12. The extended moment map

$$
\begin{aligned}
& \hat{J}^{\mathcal{D}_{A}}: T^{*} \widetilde{G} \longrightarrow \operatorname{diff}_{1}^{\wedge}(0,-l, 0) * \\
& \hat{J}^{\mathcal{D}_{A}}:(g, \mu) \longmapsto\left(J^{\mathcal{D}_{A}}, 1\right),
\end{aligned}
$$

which also generates the $\mathcal{D}_{1} \ltimes \mathcal{D}_{0}^{+}$-action (1.95), is equivariant with respect to the extended coadjoint action (1.106). It is thus a Poisson map with respect to the Lie Poisson structure determined by the Poisson brackets

$$
\begin{aligned}
\left.\left\{\left\langle\cdot \mid\left(\alpha_{1}, \beta_{1}, b_{1}\right)\right\rangle_{\mathcal{D}},\left\langle\cdot \mid\left(\alpha_{2}, \beta_{2}, b_{2}\right)\right\rangle_{\mathcal{D}}\right\}\right|_{(v, w, a)}= & \left\langle v, \alpha_{1} \alpha_{2}^{\prime}-\alpha_{2} \alpha_{1}^{\prime}\right\rangle+\left\langle w, \alpha_{1} \beta_{2}^{\prime}-\alpha_{2} \beta_{1}^{\prime}\right\rangle \\
& -a l\left(\left\langle\alpha_{1}^{\prime}, \beta_{2}^{\prime}\right\rangle-\left\langle\alpha_{2}^{\prime}, \beta_{1}^{\prime}\right\rangle\right) .
\end{aligned}
$$

From formulae $(1.98 \mathrm{~b}, \mathrm{c})$, we see that the moment map $\hat{J}^{\mathcal{D}_{A}}: T^{*} \widetilde{G} \rightarrow \operatorname{diff}_{1}^{\wedge}(0 l 0) *$ factors through $\hat{J}^{L A}: T^{*} \widetilde{G} \rightarrow\left(\widetilde{\mathfrak{g}} \dot{+} \widetilde{\mathfrak{g}}_{A}\right)^{\wedge *}$, suggesting again that the $\mathcal{D}_{1} \ltimes \mathcal{D}_{0}^{+}$-action (1.95) induces a Hamiltonian action on $\left(\widetilde{\mathfrak{g}} \dot{+} \widetilde{\mathfrak{g}}_{A}\right)^{\wedge *}$. This is easily computed, and the result is summarized as follows. (Eqs. $(1.110 \mathrm{~b}, \mathrm{c})$ for the case $\theta_{0}=-\theta_{1}=\theta$ will reappear as eqs. (2.42a,b) in Section 2.)

Proposition 1.13. The moment map $\hat{J}^{L A}: T^{*} \widetilde{G} \rightarrow\left(\mathfrak{g} \dot{+} \mathfrak{g}_{A}\right)^{\wedge *}$ is equivariant with respect to the $\mathcal{D}_{1} \ltimes \mathcal{D}_{0}^{+}$action (1.95) on $T^{*} \widetilde{G}$ and the following action on $\left(\mathfrak{g} \dot{+} \mathfrak{g}_{A}\right)^{\wedge *}$ :

$$
\begin{aligned}
(\widetilde{\sigma}, f):\left(\widetilde{\mathfrak{g}} \dot{+} \widetilde{\mathfrak{g}}_{A}\right)^{\wedge *} \longrightarrow\left(\widetilde{\mathfrak{g}} \dot{+} \widetilde{\mathfrak{g}}_{A}\right)^{\wedge *} & \\
(\widetilde{\sigma}, f):(U, V, a) & \longmapsto(\check{U}, \check{V}, a),
\end{aligned}
$$


where

$$
\begin{aligned}
& \check{U}=\widetilde{\sigma} \Theta_{1}\left(\widetilde{\sigma}^{\prime}\right)(U \circ \widetilde{\sigma}) \Theta_{1}\left(\widetilde{\sigma}^{\prime}\right)^{-1}+\frac{\widetilde{\sigma}^{\prime \prime}}{\widetilde{\sigma}^{\prime}} \theta_{1} \\
& \check{V}=\widetilde{\sigma}^{\prime} \Theta_{1}\left(\widetilde{\sigma}^{\prime}\right)\left[\left(V \circ \widetilde{\sigma}+\ln (f \circ \widetilde{\sigma})\left[\theta_{0}, V \circ \widetilde{\sigma}\right]\right] \Theta_{1}\left(\widetilde{\sigma}^{\prime}\right)^{-1}+\frac{(f \circ \widetilde{\sigma})^{\prime}}{f \circ \widetilde{\sigma}} \theta_{0} .\right.
\end{aligned}
$$

This action is Hamiltonian, and generated by the equivariant moment map

$$
\begin{aligned}
\hat{J}^{d_{A}}:\left(\widetilde{\mathfrak{g}} \dot{+} \widetilde{\mathfrak{g}}_{A}\right)^{\wedge *} & \longrightarrow \operatorname{diff}_{1}^{\wedge(0,-l, 0) *} \\
\hat{J}^{d_{A}}:(U, V, a) & \longmapsto\left(J^{d 1_{A}}, J^{d 0_{A}}, 1\right),
\end{aligned}
$$

where

$$
\begin{aligned}
J^{d 0_{A}} & :=\mathcal{B}\left(U, \theta_{0}\right) \\
J^{\alpha 1_{A}} & :=\frac{1}{a}\left[-\mathcal{B}\left(V^{\prime}, \theta_{1}\right)+\mathcal{B}(U, V)\right] .
\end{aligned}
$$

This is therefore a Poisson map with respect to the Lie Poisson structure determined by the Poisson brackets (1.108).

Proof. Differentiating along the flow generated by the action (1.109a-c) corresponding to the 1-parameter group $\{\exp [-t(\alpha, \beta)]\}$ gives the representation

$$
\begin{aligned}
X^{d_{A}}: \operatorname{diff}_{1} & \longrightarrow \chi\left(\left(\widetilde{\mathfrak{g}} \dot{+} \widetilde{\mathfrak{g}}_{A}\right)^{\wedge *}\right) \\
X^{d_{A}}:(\alpha, \beta) & \longmapsto X_{(\alpha, \beta)}^{d_{A}}:=X_{\alpha}^{d 1_{A}}+X_{\beta}^{d 0_{A}}
\end{aligned}
$$

of diff 1 by functional derivations (vector fields) on $\left(\mathfrak{g} \dot{+} \mathfrak{g}_{A}\right)^{\wedge *}$ defined by:

$$
\begin{aligned}
& X_{\beta}^{d 0_{A}}:=-\left\langle\left(\beta\left[\theta_{0}, U\right]+\beta^{\prime} \theta_{0}\right), \frac{\delta}{\delta V}\right\rangle \\
& X_{\alpha}^{d 1_{A}}:=-\left\langle(\alpha V)^{\prime}+\alpha^{\prime}\left[\theta_{1}, V\right], \frac{\delta}{\delta V}\right\rangle-\left\langle(\alpha U)^{\prime}+\alpha^{\prime}\left[\theta_{1}, U\right]+\alpha^{\prime \prime} \theta_{1}, \frac{\delta}{\delta U}\right\rangle .
\end{aligned}
$$

By explicit evaluation, and use of the Poisson bracket relations (1.40a-c) and the Leibnitz rule, we find

$$
\begin{aligned}
\left\{J_{\beta}^{d 0_{A}},\langle\cdot \mid(X, Y, b)\rangle\right\} & =X_{\beta}^{d 0_{A}}(\langle\cdot \mid(X, Y, b)\rangle) \\
\left\{J_{\alpha}^{d 1_{A}},\langle\cdot \mid(X, Y, b)\rangle\right\} & =X_{\alpha}^{d 1_{A}}(\langle\cdot \mid(X, Y, b)\rangle) \\
\left\{J_{\beta_{1}}^{d 0_{A}}, J_{\beta_{2}}^{d 0_{A}}\right\} & =X_{\beta_{1}}^{d 0_{A}}\left(J_{\beta_{2}}^{d 0_{A}}\right)=0 \\
\left\{J_{\alpha}^{d 1_{A}}, J_{\beta}^{d 0_{A}}\right\} & =-X_{\beta}^{d 0_{A}}\left(J_{\alpha}^{d 1_{A}}\right)=J_{\alpha \beta^{\prime}}^{d 0_{A}}+\mathcal{B}\left(\theta_{0}, \theta_{1}\right)\left\langle\alpha^{\prime}, \beta^{\prime}\right\rangle \\
\left\{J_{\alpha_{1}}^{d 1_{A}}, J_{\alpha_{2}}^{d 1_{A}}\right\} & =X_{\alpha_{1}}^{d 1_{A}}\left(J_{\alpha_{2}}^{d 1_{A}}\right)=J_{\left(\alpha_{1} \alpha_{2}^{\prime}-\alpha_{2} \alpha_{1}^{\prime}\right)}^{d 1_{A}},
\end{aligned}
$$


showing both that $\hat{J}^{d_{A}}=\left(J^{d 1_{A}}, J^{d 0_{A}}, 1\right)$ is the moment map generating the action $(1.109 \mathrm{a}-\mathrm{c})$, and that it is equivariant, hence preserving the respective Lie Poisson structures on $\left(\widetilde{\mathfrak{g}} \dot{+} \widetilde{\mathfrak{g}}_{A}\right)^{\wedge *}$ and $\operatorname{diff}_{1}^{\wedge(0,-l, 0) *}$ defined by eqs. (1.39) and (1.108).

For the applications to integrable systems to be discussed in Section 2, a particular case of the $\mathcal{D}_{1} \ltimes \mathcal{D}_{0}^{+}$action (1.109a-c) and moment map (1.110a-c) will be used; namely, when

$$
\theta_{0}=-\theta_{1}=: \theta
$$

and hence

$$
\mathcal{B}(\theta, \theta)=l
$$

Finally, combining the results of Propositions 1.11 and 1.13 and Corollary 1.12, we get:

Theorem 1.14. For $k=0$, the moment map $\hat{J}^{\mathcal{D}_{A}}: T^{*} \widetilde{G} \rightarrow$ diff $_{1}^{\wedge(0,-l, 0) *}$ factors into

$$
\hat{J}^{\mathcal{D}_{A}}=\hat{J}^{d_{A}} \circ \hat{J}^{L A}
$$

giving the following commuting diagram of equivariant moment maps:

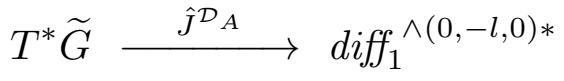

$$
\begin{aligned}
& \hat{J}^{L A} \searrow \quad \nearrow \hat{J}^{d} \\
& \left(\widetilde{\mathfrak{g}}+\widetilde{\mathfrak{g}}_{A}\right)^{\wedge *}
\end{aligned}
$$

\section{Integrable Systems}

2a. Algebraic Language

The preceding section emphasized the symplectic geometry of $T^{*} \widetilde{G}$ and Lie Poisson structures. In modern theories of integrable systems in $1+1$ dimensions, one often deals with local evolution equations, for which the language of differential algebra is the most efficient. On a practical level, the translation from functional to algebraic language consists of replacing: 
1) Functionals by their densities, and integration by parts by equivalence modulo "divergences" for these densities. (The algebraic calculus of variation results thereby.)

2) Symplectic forms and Poisson brackets by a Hamiltonian map $H \mapsto X_{H}$ assigning to any Hamiltonian density $H$ the evolution derivation $X_{H}$ via the rule

$$
\left(\boldsymbol{q}_{, t}=\right) X_{H}(\boldsymbol{q})=B\left(\frac{\delta H}{\delta \boldsymbol{q}}\right),
$$

where $\boldsymbol{q}$ is a column-vector of the basic variables ("fields") chosen in a fixed basis, with $i^{\text {th }}$ component $q_{i} ; \frac{\delta H}{\delta \boldsymbol{q}}$ is the column-vector of variational derivatives of $H$, and $B$ is a skew symmetric matrix differential operator whose properties guarantee the Jacobi identity.

In the loop space setting, the most frequently met structure consists of Lie Poisson brackets associated with centrally extended Lie algebras. This is translated into the following algebraic construction. Denote by $K$ a differential algebra with derivation $\partial$. (In Section 1, these were $K=C^{\infty}(\mathbb{R})$ or $C^{\infty}\left(S^{1}\right)$ and $\partial=\partial / \partial \sigma$.) Let $\mathcal{G}=K^{\bar{n}}$, $\bar{n} \in \mathbf{N} \cup\{\infty\}$, be a differential Lie algebra consisting of column vectors of dimension $\bar{n}$ with entries in $K$. The commutator in $\mathcal{G}$ is of the form:

$$
\begin{aligned}
{[X, Y]_{k} } & =\sum_{i j p r} c_{i j p r}^{k} \partial^{p}\left(X_{i}\right) \partial^{r}\left(Y_{j}\right) \quad(\text { finite sum } \forall k), \\
X, Y & \in \mathcal{G},
\end{aligned}
$$

where $c_{i j p r}^{k} \in K$ are structure elements defining $\mathcal{G}$ and $X_{i} \in K$ denotes the $i^{\text {th }}$ component of $X \in K^{\bar{n}}$. Let $\Omega$ be a (generalized) 2-cocycle on $\mathcal{G}$. This means that $\Omega: \mathcal{G} \times \mathcal{G} \rightarrow K$ is a bilinear skewsymmetric differential operator satisfying

$$
\begin{gathered}
\Omega([X, Y], Z)+\Omega([Y, Z], X)+\Omega([Z, X], Y) \sim 0 \\
\Omega(X, Y) \sim-\Omega(Y, X), \\
\forall X, Y, Z \in \mathcal{G},
\end{gathered}
$$

where $(\cdot) \sim 0$ means that $(\cdot) \in \operatorname{Im} \partial$. In the loop algebra setting, one associates with this data the centrally extended algebra $\mathcal{G}^{\wedge}=\mathcal{G} \oplus \mathbb{R}$, with Lie bracket

$$
[(X, a),(Y, b)]=\left([X, Y], \int \Omega(X, Y) d \sigma\right), \quad X, Y \in \mathcal{G}, \quad a, b \in \mathbb{R} .
$$


In the algebraic approach, one associates to the pair $(\mathcal{G}, \Omega)$ an affine Hamiltonian matrix (of differential operators)

$$
B=B(\mathcal{G})+b_{\Omega}
$$

where the linear part $B(\mathcal{G})$ (in the basic variables $\boldsymbol{q}$ ) of the Hamiltonian matrix $B$ is defined by the relation

$$
[B(\mathcal{G})(X)]^{t} Y \sim \boldsymbol{q}^{t}[X, Y]:=\sum_{k=1}^{\bar{n}} q_{k}[X, Y]_{k}, \quad \forall X, Y \in \mathcal{G} .
$$

A $\boldsymbol{q}$-independent matrix differential operator $b_{\Omega}$ is associated to the bilinear form $\Omega$ via the rule

$$
\left[b_{\Omega}(X)\right]^{t} Y \sim \Omega(X, Y), \quad \forall X, Y \in \mathcal{G}
$$

One of the basic features of the algebraic Hamiltonian formalism is that there is a natural one-to-one correspondence, given by the formulae (2.6a,b), between pairs $(\mathcal{G}, \Omega)$ and affine Hamiltonian matrices. (The latter encompass more general situations than the geometric ones, where the Hamiltonian operators are of order zero in $\partial$. For example, Lie algebras of differential and pseudodifferential operators have no natural local description as the infinitesimal form of groups.) The geometric Lie Poisson brackets of the previous section, which express the multiplication rule (2.4) in the language of linear functionals can be extracted from formula (2.5) via the following computation. Let $X, Y \in \mathcal{G}$, and let $H=\boldsymbol{q}^{t} X, F=\boldsymbol{q}^{t} Y$ be linear Hamiltonians. Then

$$
\begin{aligned}
\left\{\boldsymbol{q}^{t} X, \boldsymbol{q}^{t} Y\right\} & =\{H, F\}=: X_{H}(F) \sim \frac{\delta F}{\delta \boldsymbol{q}^{t}} X_{H}(\boldsymbol{q})=\frac{\delta F}{\delta \boldsymbol{q}^{t}} B\left(\frac{\delta H}{\delta \boldsymbol{q}}\right) \\
& =Y^{t}\left[B(\mathcal{G})+b_{\Omega}\right](X) \sim \boldsymbol{q}^{t}[X, Y]+\Omega(X, Y) .
\end{aligned}
$$

Remark 2.1. The recipe (2.5), (2.6a,b) handles situations, like that of formula (2.4), where the centrally extended Lie algebra in question has components of different differential dimension; $X$ and $Y$ are functions, $a$ and $b$ are numbers. When $\mathcal{G}$ is a Lie algebra over $\mathbb{R}$ (or $\mathbb{C}$, etc.) rather than over $K$, and $\Omega$ is a true 2 -cocycle, not a generalized one (i.e. one has an equality sign instead of the $\sim \operatorname{sign}$ in formulae (2.2), (2.3)), then $\mathcal{G}^{\wedge} \sim \mathcal{G} \oplus \mathbb{R}$ is again a Lie algebra over $\mathbb{R}$. Treating it as a new Lie algebra, we get from formulae $(2.6 \mathrm{a}, \mathrm{b})$ that

$$
B\left(\mathcal{G}^{\wedge}\right)=\begin{array}{c|c}
\boldsymbol{q} & a \\
a
\end{array}\left(\begin{array}{c|c}
B(\mathcal{G})+a b_{\Omega} & \mathbf{0} \\
\hline \mathbf{0}^{t} & 0
\end{array}\right),
$$


where $a$ is the extra coordinate on the $\mathbb{R}$-part of $\mathcal{G}^{\wedge *} \sim \mathcal{G}^{*} \oplus \mathbb{R}$. Thus, we can let

$$
a=\text { const. }
$$

in formula (2.8) and what remains from $B\left(\mathcal{G}^{\wedge}\right)$ is our universal formula $(2.5)$ (for $a=1$ ).

Let us consider, as an illustration, the case of the Lie algebra $\widetilde{\mathfrak{g}}$ (or $L \mathfrak{g}$ ) of the previous section. Fix a basis in $\mathfrak{g}$. Then $\widetilde{\mathfrak{g}}=K^{\bar{n}}$, where $\bar{n}=\operatorname{dimg}$. Let the Ad-invariant form $\mathcal{B}$ on $\mathfrak{g}$ be given, in the chosen basis, by a symmetric matrix $\overline{\mathcal{B}}$ :

$$
\mathcal{B}(x, y)=y^{t} \overline{\mathcal{B}} x, \quad x, y \in \mathfrak{g}
$$

and let $c_{i j}^{k}$ be the structure constants of $\mathfrak{g}$ in the same basis:

$$
[x, y]_{k}=\sum_{j k} c_{i j}^{k} x_{j} y_{i}
$$

Then, for both $\mathfrak{g}($ over $\mathbb{R})$ and $\widetilde{\mathfrak{g}}($ over $K$ )

$$
B(\mathfrak{g})_{i j}=B(\widetilde{\mathfrak{g}})_{i j}=\sum_{k j} c_{i j}^{k} q_{k}
$$

Also, since

$$
\Omega(X, Y)=\mathcal{B}(\partial(X), Y)=Y^{t} \overline{\mathcal{B}} \partial(X)
$$

we see that

$$
b_{\Omega}=\overline{\mathcal{B}} \partial
$$

so that, finally,

$$
B_{i j}=\sum c_{i j}^{k} q_{k}+\overline{\mathcal{B}}_{i j} \partial
$$

If an orthonormal basis for $\mathfrak{g}$ is chosen (with respect to $\mathcal{B}$ ), we have

$$
\overline{\mathcal{B}}_{i j}=\delta_{i j} \quad, \quad c_{i j}^{k}=c_{j k}^{i}
$$

so that the equations of motion for a Hamiltonian $H$ are

$$
\dot{q}_{i}=\sum_{j} B_{i j}\left(\frac{\delta H}{\delta q_{j}}\right)=\sum_{j k} c_{i j}^{k} q_{k} \frac{\delta H}{\delta q_{j}}+\left(\frac{\delta H}{\delta q_{i}}\right)^{\prime}=-\left[\boldsymbol{q}, \frac{\delta H}{\delta \boldsymbol{q}}\right]_{i}+\left[\left(\frac{\delta H}{\delta \boldsymbol{q}}\right)^{\prime}\right]_{i}
$$


i.e.

$$
\dot{\boldsymbol{q}}=(-[\boldsymbol{q}, \quad]+\partial \mathbf{1})\left(\frac{\delta H}{\delta \boldsymbol{q}}\right) .
$$

In other words,

$$
B=-[\boldsymbol{q}, \quad]+\partial \mathbf{1} .
$$

If the chosen basis of $\mathfrak{g}$ is not orthonormal, then one has the familiar form

$$
B(\cdot)=-a d_{(\cdot,)}^{*}+\overline{\mathcal{B}} \partial .
$$

Finally, we discuss Hamiltonian maps. Suppose $B$ is a Hamiltonian matrix over the ring $C_{q}=K\left[\boldsymbol{q}, \boldsymbol{q}^{\prime}, \ldots\right]$, and let $B_{1}$ be a Hamiltonian matrix over another $\operatorname{ring} C_{u}$. A homomorphism of differential rings (over $K$ )

$$
\Phi: C_{u} \rightarrow C_{q}
$$

which commutes with $\partial$ is called a Hamiltonian map if

$$
\Phi X_{H}=X_{\Phi(H)} \Phi, \quad \forall H \in C_{u}
$$

(This map should be viewed as dual to a Poisson map on the fields $\boldsymbol{q} \mapsto \boldsymbol{u}$ which was the object of study throughout Section 1.) In terms of the Hamiltonian matrices $B$ and $B_{1}$, the compatibility condition (2.21) is expressed by the equality

$$
\Phi\left(B_{1}\right)=D(\boldsymbol{\Phi}) B D(\boldsymbol{\Phi})^{\dagger},
$$

where:

$$
\Phi:=\Phi(\boldsymbol{u}), \quad \Phi_{\alpha}=\Phi\left(u_{\alpha}\right),
$$

$D(\boldsymbol{\Phi})$ is the Fréchet derivative of $\boldsymbol{\Phi}$ :

$$
D(\Phi)_{\alpha i}=\sum_{\ell} \frac{\partial \Phi_{\alpha}}{\partial q_{i}^{(\ell)}} \partial^{\ell},
$$

and '†' denotes adjoint.

As an example,

$$
2 B_{1}=2(u \partial+\partial u)-l \partial^{3}
$$


defines the differential algebraic version of the Virasoro algebra, denoted here $C_{u}$ (equiv. $\left(\text { diff } S^{1}\right)^{\wedge}$ ) associated with the generalized 2-cocycle

$$
\Omega\left(\alpha_{1}, \alpha_{2}\right)=\frac{l}{2} \alpha_{1} \partial^{3}\left(\alpha_{2}\right)
$$

on the Lie algebra of vector fields on $\mathbb{R}\left(\right.$ or $\left.S^{1}\right)$.

Consider the ring homomorphism generated by

$$
\Phi(u)=\boldsymbol{\theta}^{t} \boldsymbol{q}^{\prime}+\boldsymbol{q}^{t} \boldsymbol{q}
$$

from $C_{u}$ into $C_{q}$, where $\boldsymbol{\theta}$ is a fixed (constant) vector in $\mathfrak{g}$, with the Hamiltonian matrix $B$ in the ring $C_{q}$ given by formula (2.18). Then $\Phi$ is a Hamiltonian map, since

$$
\begin{aligned}
D(\boldsymbol{\Phi}) B D(\boldsymbol{\Phi})^{\dagger} & =\left(\boldsymbol{\theta}^{t} \partial+2 \boldsymbol{q}^{t}\right)([-\boldsymbol{q}, \quad]+\partial \mathbf{1})(-\boldsymbol{\theta} \partial+2 \boldsymbol{q}) \\
& =\left(-\partial \boldsymbol{\theta}^{t}[\boldsymbol{q}, \quad]+\boldsymbol{\theta}^{t} \partial^{2}+2 \boldsymbol{q}^{t} \partial\right)(-\boldsymbol{\theta} \partial+2 \boldsymbol{q}) \\
& =-\boldsymbol{\theta}^{t} \boldsymbol{\theta} \partial^{3}+2 \partial^{2} \boldsymbol{\theta}^{t} \boldsymbol{q}-2 \boldsymbol{q}^{t} \boldsymbol{\theta} \partial^{2}+4 \boldsymbol{q}^{t} \partial \boldsymbol{q} \\
& =-\boldsymbol{\theta}^{t} \boldsymbol{\theta} \partial^{3}+2\left(\boldsymbol{\theta}^{t} \boldsymbol{q}^{\prime} \partial+\partial \boldsymbol{\theta}^{t} \boldsymbol{q}^{\prime}\right)+2\left(\boldsymbol{q}^{t} \boldsymbol{q} \partial+\partial \boldsymbol{q}^{t} \boldsymbol{q}\right) \\
& =\Phi\left(-\boldsymbol{\theta}^{t} \boldsymbol{\theta} \partial^{3}+2 u \partial+2 \partial u\right)=\Phi\left(2 B_{1}\right),
\end{aligned}
$$

provided

$$
l=\boldsymbol{\theta}^{t} \boldsymbol{\theta} .
$$

Thus, the criterion (2.22) is satisfied. (The geometrical significance of the Hamiltonian map (2.27) in the loop group setting is explained in $\mathbf{H K 1 ] . )}$

Remark 2.2. To make contact with the notation of the previous section, we adopt the convention that boldface letters denote column vectors representing the components of elements either of $\mathfrak{g}$ relative to some basis, or of $\mathfrak{g}^{*}$ relative to the dual basis, or elements of the corresponding loop algebra $\widetilde{\mathfrak{g}}$ or its dual $\widetilde{\mathfrak{g}}^{*}$. Thus, the elements $\theta, \phi \in \mathfrak{g}$ are replaced by the column vectors $\boldsymbol{\theta}, \boldsymbol{\phi} \in \mathbb{R}^{\operatorname{dimg}}$, while the elements $U, V \in \widetilde{\mathfrak{g}}^{*}$ are replaced by the column vectors $\mathbf{U}, \mathbf{V} \in K^{\operatorname{dimg}}$.

\section{2b. Dispersive Wave Systems}

In Section 1 we derived two families of Poisson maps:

$$
\hat{J}^{d}:(\widetilde{\mathfrak{g}} \oplus \widetilde{\mathfrak{g}})^{\wedge *} \longrightarrow \operatorname{diff}_{1}^{\wedge\left(l_{1} l_{2} l_{3}\right) *},
$$


defined by eqs. $(1.82 \mathrm{a}-\mathrm{c})$ and

$$
\hat{J}^{d_{A}}:\left(\widetilde{\mathfrak{g}} \dot{+} \widetilde{\mathfrak{g}}_{A}\right)^{\wedge *} \longrightarrow \operatorname{diff}_{1}^{\wedge(0,-l, 0) *},
$$

defined by eqs. (1.91a-c). In the following, we shall consider two special cases of these maps; for the map (2.30), we take the values for $\theta_{0}, \theta_{1}, \phi_{0}, \phi_{1}$ in eqs. (1.82a-c) as given by eqs. $(1.78 \mathrm{a}, \mathrm{b})$, for which $\left(l_{1} l_{2} l_{3}\right)=(4 l,-2 l, 0)$, and denote the corresponding centrally extended algebra $\operatorname{diff}_{1(1)}:=\operatorname{diff}_{1}^{\wedge(4 l,-2 l, 0)}$ for brevity. For the map (2.31), we choose the values for $\theta_{0}, \theta_{1}$ in eq. (1.91a-c) as given by eqs. (1.113a) and denote the centrally extended algebra $\operatorname{diff}_{1(0)}^{\wedge}:=\operatorname{diff}_{1}^{\wedge(0,-l, 0)}$. Thus, the subscript $(\alpha)=$ (1), (0) distinguishes two different central extensions of the Lie algebra diff 1 . We first convert these maps, according to the lexicon of the preceding subsection, into algebraic Hamiltonian maps and then derive integrable hierarchies on $(\widetilde{\mathfrak{g}} \oplus \widetilde{\mathfrak{g}})^{\wedge *}$ and $\left(\widetilde{\mathfrak{g}} \dot{+} \widetilde{\mathfrak{g}}_{A}\right)^{\wedge *}$ from known integrable hierarchies on $\operatorname{diff}_{1(\alpha)} \wedge^{*}$.

When $(\alpha)=(1)$, we have the Lie algebra $\widetilde{\mathfrak{g}} \oplus \widetilde{\mathfrak{g}}$ where, choosing normalizations to correspond to the fluid dynamical conventions in $[\mathbf{K u} \mathbf{1}]$, the commutator is

$$
\left[\left(\begin{array}{c}
\bar{X}_{1} \\
Y_{1}
\end{array}\right),\left(\begin{array}{c}
\bar{X}_{2} \\
Y_{2}
\end{array}\right)\right]=\left(\begin{array}{c}
2\left[\bar{X}_{1}, \bar{X}_{2}\right] \\
{\left[Y_{1}, Y_{2}\right]}
\end{array}\right), \quad \bar{X}_{i}, Y_{i} \in \widetilde{\mathfrak{g}},
$$

and the 2 -cocycle is

$$
\Omega(1,2):=\Omega\left(\left(\begin{array}{c}
\bar{X}_{1} \\
Y_{1}
\end{array}\right),\left(\begin{array}{c}
\bar{X}_{2} \\
Y_{2}
\end{array}\right)\right)=2 \bar{X}_{1}^{t} \bar{X}_{2}^{\prime}-\frac{1}{2} Y_{1}^{t} Y_{2}^{\prime}
$$

Thus, we are working with the centrally extended algebra $(\widetilde{\mathfrak{g}} \oplus \widetilde{\mathfrak{g}})^{\wedge}$, with Lie bracket (1.20), under the identifications $\left(\frac{1}{2} X, Y\right)=(\bar{X}, Y)$ and the 2-cocycle $\Omega$ of eq. (2.32b) is normalized to half $c^{L R}$ of eq. (1.19). To simplify calculations, we choose from now on an orthonormal basis in $\mathfrak{g}$. Denoting the generic basic variable $\boldsymbol{q}$ by $\mathbf{U}$ and $\overline{\mathbf{V}}$ (corresponding to $2 U$ and $V$ in (1.21)), the Hamiltonian matrix (2.5) encoding the data $(2.32 \mathrm{a}, \mathrm{b})$ is

$$
B_{(1)}=\left(\begin{array}{cc}
-2[\mathbf{U},]+2 \partial \mathbf{1} & \mathbf{0} \\
\mathbf{0} & -[\overline{\mathbf{V}},]-\frac{1}{2} \partial \mathbf{1}
\end{array}\right)
$$


This Hamiltonian matrix is thus the algebraic version of the Lie Poisson bracket (1.24) at $a=\frac{1}{2}$.

For the Lie algebra $\operatorname{diff}_{1}$, we have the commutator

$$
\left[\left(\begin{array}{c}
\beta_{1} \\
\alpha_{1}
\end{array}\right),\left(\begin{array}{c}
\beta_{2} \\
\alpha_{2}
\end{array}\right)\right]=\left(\begin{array}{c}
\alpha_{1} \beta_{2}^{\prime}-\alpha_{2} \beta_{1}^{\prime} \\
\alpha_{1} \alpha_{2}^{\prime}-\alpha_{1}^{\prime} \alpha_{2}
\end{array}\right), \quad \beta_{i}, \alpha_{i} \in K
$$

and the 2-cocycle

$$
\Omega_{(1)}(1,2)=l\left(2 \beta_{1} \beta_{2}^{\prime}+\alpha_{1} \beta_{2}^{\prime \prime}-\beta_{1} \alpha_{2}^{\prime \prime}\right)
$$

corresponding to the central extension $\operatorname{diff}_{1(1)}($ cf. eq. $(1.78 \mathrm{c}))$. Denoting the generic basic variable $\boldsymbol{q}$ as $h:=v$ and $u:=w$ (the standard notation from fluid dynamics), the corresponding Hamiltonian matrix is

$$
\bar{B}_{(1)}=\left(\begin{array}{cc}
2 l \partial & \partial u-l \partial^{2} \\
u \partial+l \partial^{2} & h \partial+\partial h
\end{array}\right)
$$

This gives the algebraic counterpart of the Lie Poisson bracket (1.79) (at $\left.a=\frac{1}{2}\right)$.

For $\operatorname{diff}_{1(0)}^{\wedge}$, we have another 2-cocycle on the Lie algebra $\operatorname{diff}_{1}$ :

$$
\Omega_{(0)}(1,2)=l\left(\alpha_{1} \beta_{2}^{\prime \prime}-\beta_{1} \alpha_{2}^{\prime \prime}\right)
$$

The corresponding Hamiltonian matrix on $\operatorname{diff}_{1(0)}^{\wedge *}$ is

$$
\bar{B}_{(0)}=\left(\begin{array}{cc}
0 & \partial u-l \partial^{2} \\
u \partial+l \partial^{2} & h \partial+\partial h
\end{array}\right)
$$

which is the counterpart of the Lie Poisson bracket (1.108) at $a=1$.

For the Lie algebra $\widetilde{\mathfrak{g}}+\widetilde{\mathfrak{g}}_{A}$, we have the commutator (cf. (1.26))

$$
\left[\left(\begin{array}{c}
Y_{1} \\
X_{1}
\end{array}\right)\left(\begin{array}{c}
Y_{2} \\
X_{2}
\end{array}\right)\right]=\left(\begin{array}{c}
{\left[X_{1}, Y_{2}\right]-\left[X_{2}, Y_{1}\right]} \\
{\left[X_{1}, X_{2}\right]}
\end{array}\right), \quad X_{i}, Y_{i} \in \widetilde{\mathfrak{g}}
$$

and the 2-cocycle (cf. (1.37))

$$
\Omega(1,2)=Y_{1}^{t} X_{2}^{\prime}+X_{1}^{t} Y_{2}^{\prime}
$$


so that the corresponding Hamiltonian matrix, in the variables $\boldsymbol{q}=(\mathbf{U}, \mathbf{V}$ ) (which correspond in this case to the pair $(U, V)$ in $(1.27))$, is

$$
B_{(0)}=\left(\begin{array}{cc}
\mathbf{0} & -[\mathbf{U},]+\partial \mathbf{1} \\
-[\mathbf{U},]+\partial \mathbf{1} & -[\mathbf{V},]
\end{array}\right),
$$

which is the algebraic counterpart of the Lie Poisson bracket (1.47) at $a=1$.

From Theorem 1.9 of the preceding section, with $\phi_{0}=0, \theta_{0}=-2 \phi_{1}=-2 \theta_{1}=2 \theta$, $a=\frac{1}{2}$, and $\theta$ replaced by the corresponding column vector $\boldsymbol{\theta}$, we have

Proposition 2.1. The map $\Phi_{(1)}: C_{u, h} \rightarrow C_{\mathbf{U}, \overline{\mathbf{V}}}$, given on the generators $u$ and $h$ by the formulae

$$
\begin{aligned}
& \Phi_{(1)}(u)=\boldsymbol{\theta}^{t} \mathbf{U} \\
& \Phi_{(1)}(h)=\boldsymbol{\theta}^{t}\left(\frac{1}{2} \mathbf{U}^{\prime}+\overline{\mathbf{V}}\right)^{\prime}+\frac{1}{4} \mathbf{U}^{t} \mathbf{U}-\overline{\mathbf{V}}^{t} \overline{\mathbf{V}}
\end{aligned}
$$

is a Hamiltonian map with respect to the Hamiltonian matrices $\bar{B}_{(1)}$ (2.36) and $B_{(1)}$ (2.33), with $l:=\boldsymbol{\theta}^{t} \boldsymbol{\theta}$.

From Proposition 1.13 of the preceding section, with $\theta=\theta_{0}=-\theta_{1}$, we have

Proposition 2.2. The map $\Phi_{(0)}: C_{u, h} \rightarrow C_{\mathbf{U}, \mathbf{V}}$, defined by the formulae

$$
\begin{aligned}
& \Phi_{(0)}(u)=\boldsymbol{\theta}^{t} \mathbf{U} \\
& \Phi_{(0)}(h)=\boldsymbol{\theta}^{t} \mathbf{V}^{\prime}+\mathbf{U}^{t} \mathbf{V},
\end{aligned}
$$

is a Hamiltonian map with respect to the Hamiltonian matrices $\bar{B}_{(0)}$ (2.37) and $B_{(0)}$ (2.41), again with $l:=\boldsymbol{\theta}^{t} \boldsymbol{\theta}$.

These results may also be verified directly, of course, by a computation similar to (2.28). Until the end of this section, $\boldsymbol{\theta}$ will be taken to have unit length:

$$
l=\boldsymbol{\theta}^{t} \boldsymbol{\theta}=1 .
$$

In the Hamiltonian structure $\bar{B}_{(1)}(2.36)$, the following sequence of Hamiltonians

$$
\begin{aligned}
& H_{1}=h, \quad H_{2}=u h, \quad \ldots \\
& H_{n}:=\frac{1}{n} \operatorname{Res}\left(\partial+u+h \partial^{-1}\right)^{n}, \quad n \in \mathbf{N},
\end{aligned}
$$


where

$$
\operatorname{Res}\left(\sum a_{i} \partial^{i}\right):=a_{-1},
$$

is known to form a commuting family [Ku1]. The first flow, with $H=h$, produces the $\sigma$-shift (i.e. $\dot{\boldsymbol{q}}=\boldsymbol{q}^{\prime}$, whatever $\boldsymbol{q}$ is), while the flow of the Hamiltonian

$$
H=\frac{1}{2} H_{2}=\frac{1}{2} u h
$$

produces the equations of dispersive water waves (DWW)

$$
\begin{aligned}
& \dot{u}=\left(\frac{1}{2} u^{2}+h-\frac{1}{2} u^{\prime}\right)^{\prime} \\
& \dot{h}=\left(u h+\frac{1}{2} h^{\prime}\right)^{\prime} .
\end{aligned}
$$

When $\mathfrak{g}$ is one-dimensional, the Hamiltonian map $\Phi_{(1)}$ produces an infinite commuting hierarchy of Hamiltonians $\Phi_{(1)}\left(H_{n}\right)$, called the modified dispersive water wave (mDWW) hierarchy (in the variables $U, \bar{V}+\frac{1}{2} U$ ). For $\mathfrak{g}$ arbitrary, we again obtain an infinite commuting hierarchy, this time with respect to the Hamiltonian structure (2.33). The first flow, with Hamiltonian

$$
\Phi_{(1)}(h) \sim \frac{1}{4} \mathbf{U}^{t} \mathbf{U}-\overline{\mathbf{V}}^{t} \overline{\mathbf{V}},
$$

is just the $\sigma$-shift, as was to be expected.

Let us compute the equations of motion for the next flow, which we denote $\mathfrak{g}$ mDWW (g-modified dispersive water waves). We have, for any $H \in C_{u, h}$ :

$$
\left(\begin{array}{c}
\frac{\delta}{\delta \mathbf{U}} \\
\frac{\delta}{\delta \overline{\mathbf{V}}}
\end{array}\right)\left(\Phi_{(1)}(H)\right)=D\left(\boldsymbol{\Phi}_{(1)}\right)^{\dagger} \Phi_{(1)}\left(\begin{array}{c}
\frac{\delta}{\delta u} \\
\frac{\delta}{\delta h}
\end{array}\right)(H),
$$

where

$$
\Phi_{(1)}=\left(\begin{array}{c}
\Phi_{(1)}(u) \\
\Phi_{(1)}(h)
\end{array}\right)=\left(\begin{array}{c}
\boldsymbol{\theta}^{t} \mathbf{U} \\
\boldsymbol{\theta}^{t}\left(\frac{1}{2} \mathbf{U}^{\prime}+\overline{\mathbf{V}}^{\prime}\right)+\frac{1}{4} \mathbf{U}^{t} \mathbf{U}-\overline{\mathbf{V}}^{t} \overline{\mathbf{V}}
\end{array}\right)
$$

(which is the same as formula (2.42)). Hence,

$$
D\left(\boldsymbol{\Phi}_{(1)}\right)^{\dagger}=\left(\begin{array}{cc}
\boldsymbol{\theta} & -\frac{1}{2} \boldsymbol{\theta} \partial+\frac{1}{2} \mathbf{U} \\
\mathbf{0} & -\boldsymbol{\theta} \partial-2 \overline{\mathbf{V}}
\end{array}\right) .
$$


Denoting, for brevity,

$$
\tilde{u}:=\Phi_{(1)}(u), \quad \tilde{h}:=\Phi_{(1)}(h)
$$

we then get, for $H=u h / 2$ :

$$
\begin{aligned}
& \frac{\delta \Phi_{(1)}(H)}{\delta \mathbf{U}}=\left(\frac{1}{2} \tilde{h}-\frac{1}{4} \tilde{u}^{\prime}\right) \boldsymbol{\theta}+\frac{1}{4} \tilde{u} \mathbf{U}, \\
& \frac{\delta \Phi_{(1)}(H)}{\delta \overline{\mathbf{V}}}=-\frac{1}{2} \tilde{u}^{\prime} \boldsymbol{\theta}-\tilde{u} \overline{\mathbf{V}} .
\end{aligned}
$$

Applying the Hamiltonian matrix $B_{(1)}(2.33)$ to the vector $(2.54 \mathrm{a}, \mathrm{b})$, we obtain the $\mathfrak{g}-\mathrm{mDWW}$ equations of motion:

$$
\begin{aligned}
& \dot{\mathbf{U}}=\left(\frac{1}{2} \tilde{h}-\frac{1}{4} \tilde{u}^{\prime}\right)[\boldsymbol{\theta}, \mathbf{U}]+\left[\left(\tilde{h}-\frac{1}{2} \tilde{u}^{\prime}\right) \boldsymbol{\theta}+\frac{1}{2} \tilde{u} \mathbf{U}\right]^{\prime} \\
& \overline{\mathbf{V}}=\frac{1}{2} \tilde{u}^{\prime}[\overline{\mathbf{V}}, \boldsymbol{\theta}]+\left(\frac{1}{2} \tilde{u}^{\prime} \boldsymbol{\theta}+\frac{1}{2} \tilde{u} \overline{\mathbf{V}}\right)^{\prime} .
\end{aligned}
$$

Similarly, in the Hamiltonian structure $\bar{B}_{(0)}$ (2.38), the following sequence of Hamiltonians

$$
\mathcal{H}_{1}=2 h, \quad \mathcal{H}_{2}=\frac{1}{2}\left(u h+h^{2}\right), \ldots
$$

is known to form an infinite commuting family [Ku1]. The first flow, with Hamiltonian $\frac{1}{2} \mathcal{H}_{1}=h$, is the $\sigma$-shift; the second flow, with Hamiltonian $\mathcal{H}_{2}=\left(u h+h^{2}\right) / 2$, gives

$$
\begin{aligned}
& \dot{u}=\left(\frac{1}{2} u^{2}+u h-\frac{1}{2} u^{\prime}-h^{\prime}\right)^{\prime} \\
& \dot{h}=\left(u h+\frac{3}{2} h^{2}+\frac{1}{2} h^{\prime}\right)^{\prime} .
\end{aligned}
$$

which are the first mDWW equations (in the variables $(u+2 h ; h)$ ). (The reader may check that in these variables one obtains the same system as the $\{\mathfrak{g}=\mathbb{R}\}$-case of the system $(2.54 a, b)$ in the variables $\left.\left(\mathbf{U} ; \overline{\mathbf{V}}+\frac{1}{2} \mathbf{U}\right)\right)$. The Hamiltonian map $\Phi_{(0)}$ produces an infinite hierarchy of Hamiltonians $\Phi_{(0)}\left(\mathcal{H}_{n}\right)$ commuting in the Hamiltonian structure $B_{(0)}(2.41)$. For the case $\mathfrak{g}=\mathbb{R}$, this hierarchy is known as the (doubly modified) $\mathrm{m}^{2} \mathrm{DWW}$ hierarchy. Supppose now that $\mathfrak{g}$ is arbitrary. The Hamiltonian

$$
\Phi_{(0)}\left(\frac{1}{2} \mathcal{H}_{1}\right)=\Phi_{(0)}(h) \sim \mathbf{U}^{t} \mathbf{V}
$$


produces the $\sigma$-shift, as expected. To compute the first nontrivial flow, with Hamiltonian

$$
\Phi_{(0)}\left(\mathcal{H}_{2}\right)=\Phi_{0}\left[\frac{1}{2}\left(u h+h^{2}\right)\right]
$$

we have:

$$
\boldsymbol{\Phi}_{(0)}=\left(\begin{array}{c}
\Phi_{(0)}(u) \\
\Phi_{(0)}(h)
\end{array}\right)=:\left(\begin{array}{l}
\tilde{u} \\
\tilde{h}
\end{array}\right)=\left(\begin{array}{c}
\boldsymbol{\theta}^{t} \mathbf{U} \\
\boldsymbol{\theta}^{t} \mathbf{V}^{\prime}+\mathbf{U}^{t} \mathbf{V}
\end{array}\right),
$$

(which is the same as $(2.43 \mathrm{a}, \mathrm{b})$ ) so that

$$
D\left(\boldsymbol{\Phi}_{(0)}\right)^{\dagger}=\left(\begin{array}{cc}
\boldsymbol{\theta}^{t} & \mathbf{0} \\
\mathbf{V}^{t} & \mathbf{U}^{t}+\boldsymbol{\theta}^{t} \partial
\end{array}\right)^{\dagger}=\left(\begin{array}{cc}
\boldsymbol{\theta} & \mathbf{V} \\
\mathbf{0} & \mathbf{U}-\boldsymbol{\theta} \partial
\end{array}\right) .
$$

Hence, for any $H \in C_{u, h}$,

$$
\left(\begin{array}{l}
\delta / \delta \mathbf{U} \\
\delta / \delta \mathbf{V}
\end{array}\right) \Phi_{(0)}(H)=\left(\begin{array}{cc}
\boldsymbol{\theta} & \mathbf{V} \\
\mathbf{0} & \mathbf{U}-\boldsymbol{\theta} \partial
\end{array}\right) \Phi_{(0)}\left(\begin{array}{c}
\delta H / \delta u \\
\delta H / \delta h
\end{array}\right),
$$

and for the case $H=\mathcal{H}_{2}=\left(u h+h^{2}\right) / 2$, we get

$$
\left(\begin{array}{c}
\frac{\delta \Phi_{0}\left(\mathcal{H}_{2}\right)}{\delta \mathbf{U}} \\
\frac{\delta \Phi_{0}\left(\mathcal{H}_{2}\right)}{\delta \mathbf{V}}
\end{array}\right)=\left(\begin{array}{c}
\frac{1}{2} \tilde{h} \boldsymbol{\theta}+\left(\frac{1}{2} \tilde{u}+\tilde{h}\right) \mathbf{V} \\
\left(\frac{1}{2} \tilde{u}+\tilde{h}\right) \mathbf{U}-\left(\frac{1}{2} \tilde{u}+\tilde{h}\right)^{\prime} \boldsymbol{\theta}
\end{array}\right) .
$$

Applying the Hamiltonian matrix $B_{(0)}(2.41)$ to the vector (2.63), we get the first nontrivial flow in the $\mathfrak{g}-\mathrm{m}^{2} \mathrm{DWW}$ hierarchy:

$$
\begin{aligned}
\dot{\mathbf{U}} & =\left(\frac{1}{2} \tilde{u}+\tilde{h}\right)^{\prime}[\mathbf{U}, \boldsymbol{\theta}]+\left[\left(\frac{1}{2} \tilde{u}+\tilde{h}\right) \mathbf{U}-\left(\frac{1}{2} \tilde{u}+\tilde{h}\right)^{\prime} \boldsymbol{\theta}\right]^{\prime} \\
\dot{\mathbf{V}} & =\frac{1}{2} \tilde{h}[\boldsymbol{\theta}, \mathbf{U}]+\left(\frac{1}{2} \tilde{u}+\tilde{h}\right)^{\prime}[\mathbf{V}, \boldsymbol{\theta}]+\left[\frac{1}{2} \tilde{h} \boldsymbol{\theta}+\left(\frac{1}{2} \tilde{u}+\tilde{h}\right) \mathbf{V}\right]^{\prime} .
\end{aligned}
$$

Remark 2.3. There also exists a family of rational Hamiltonian maps (as opposed to the polynomial ones we have been dealing with so far $)$ from $\left(\mathfrak{g} \oplus \mathfrak{g}_{A}\right)^{\wedge *}$ into $\operatorname{diff} \hat{1}_{[\gamma]}$, $\Phi_{[\gamma]}: C_{u, h} \rightarrow C_{\mathbf{U}, \mathbf{V}}$, of the form

$$
\begin{aligned}
& \Phi_{[\gamma]}(u)=\boldsymbol{\gamma}^{t} \mathbf{U}-\epsilon \sum_{s=1}^{N}\left[\ln (\mathbf{U}+\mathbf{V})^{t} \boldsymbol{\theta}^{s}\right]^{\prime} \\
& \Phi_{[\gamma]}(h)=\frac{1}{4}\left(\mathbf{U}^{t} \mathbf{U}-\mathbf{V}^{t} \mathbf{V}\right),
\end{aligned}
$$


where $\boldsymbol{\theta}^{1}, \ldots, \boldsymbol{\theta}^{N}$ is a family of pairwise commuting constant elements in $\mathfrak{g}, \boldsymbol{\gamma}$ is another constant element in $\mathfrak{g}, \gamma:=\boldsymbol{\gamma}^{t} \boldsymbol{\gamma}$, and

$$
\begin{aligned}
B & =\left(\begin{array}{cc}
2 \partial \mathbf{1} & \mathbf{0} \\
\mathbf{0} & -4[\mathbf{V},]-2 \partial \mathbf{1}
\end{array}\right) \\
\bar{B}_{[\gamma]} & =\left(\begin{array}{cc}
2 \boldsymbol{\gamma}^{t} \boldsymbol{\gamma} \partial & \partial u-\partial^{2} \\
u \partial+\partial^{2} & h \partial+\partial h
\end{array}\right) .
\end{aligned}
$$

In particular, when $\boldsymbol{\gamma}^{t} \boldsymbol{\gamma}=1$, the Hamiltonian matrix $\bar{B}_{[1]}$ of eq. (2.66b) is the same as $\bar{B}_{(1)}$ in $(2.36)$. Therefore, the set of Hamiltonians $\left\{\Phi_{[1]}\left(H_{n}\right)\right\}$ forms a new $\mathfrak{g}-\mathrm{mDWW}$ infinite commuting hierarchy. Similarily, when $\boldsymbol{\gamma}^{t} \boldsymbol{\gamma}=0$, the Hamiltonian matrix $\bar{B}_{[0]}$ of $(2.66 \mathrm{~b})$ is the same as the Hamiltonian matrix $\bar{B}_{(0)}$ in (2.38). Hence, the set of Hamiltonians $\left\{\Phi_{[0]}\left(\mathcal{H}_{n}\right)\right\}$ forms a new $\mathfrak{g}-\mathrm{m}^{2} \mathrm{DWW}$ infinite commuting hierarchy. The geometric nature of the map $\Phi_{[\gamma]}$ defined by eqs. $(2.65 \mathrm{a}, \mathrm{b})$ is as yet a mystery.

\section{2c. Specializations}

The hierarchy of DWW equations

$$
\left(\begin{array}{l}
u \\
h
\end{array}\right)_{t}=\bar{B}_{(1)}\left(\begin{array}{c}
\delta H_{m} / \delta u \\
\delta H_{m} / \delta h
\end{array}\right), \quad m \in \mathbf{N}
$$

has, for every odd flow $m=1(\bmod 2)$, the invariant submanifold defined by $u=0$, on which this hierarchy reduces to the KdV hierarchy

$$
h_{t}=\frac{1}{2} \partial\left(\frac{\delta \bar{H}_{2 m+3}}{\delta h}\right)=\left(\frac{1}{2} \partial^{3}+h \partial+\partial h\right)\left(\frac{\delta \bar{H}_{2 m+1}}{\delta h}\right) \text {, }
$$

where

$$
\bar{H}_{m}:=\left.H_{m}\right|_{u=0} .
$$


This follows from the following formulae [Ku1]:

$$
\begin{aligned}
\frac{\delta \bar{H}_{2 m}}{\delta h} & =0 \\
\frac{\delta \bar{H}_{m+1}}{\delta h} & =\left.2 \frac{\delta H_{m}}{\delta u}\right|_{u=0}-\partial\left(\frac{\delta \bar{H}_{m}}{\delta h}\right) \\
\left.\partial\left(\frac{\delta H_{m+1}}{\delta u}\right)\right|_{u=0} & =(h \partial+\partial h)\left(\frac{\delta \bar{H}_{m}}{\delta h}\right)+\left.\partial^{2}\left(\frac{\delta H_{m}}{\delta u}\right)\right|_{u=0} \\
\partial\left(\frac{\delta \bar{H}_{m+2}}{\delta h}\right) & =\left[2(h \partial+\partial h)+\partial^{3}\right]\left(\frac{\delta \bar{H}_{m}}{\delta h}\right) \\
\left.\partial\left(\frac{\delta H_{m+2}}{\delta u}\right)\right|_{u=0} & =\left.\left[2(h \partial+\partial h)+\partial^{3}\right]\left(\frac{\delta H_{m}}{\delta u}\right)\right|_{u=0}+\left(h^{\prime} \partial+\partial h^{\prime}\right)\left(\frac{\delta \bar{H}_{m}}{\delta h}\right)_{(2.7} \\
\left(\begin{array}{c}
u \\
h
\end{array}\right)_{t} & =\bar{B}_{(1)}\left(\begin{array}{c}
\delta H_{m} / \delta u \\
\delta H_{m} / \delta h
\end{array}\right)=\left(\begin{array}{cc}
0 \\
\partial H_{m+1} / \delta u \\
\partial H_{m+1} / \delta h
\end{array}\right)
\end{aligned}
$$

Thus, when

$$
m=2 n+1, \quad n \in \mathbf{Z}_{+},
$$

from formulae (2.71), (2.70a), we get

$$
\left.u_{t}\right|_{u=0}=\left.\partial\left(\frac{\delta H_{2 n+2}}{\delta h}\right)\right|_{u=0}=0
$$

so that $\{u=0\}$ is indeed an invariant submanifold. From formulae (2.71) and (2.70b,c), we obtain

$$
\begin{aligned}
\left.h_{t}\right|_{u=0} & =\left.\partial\left(\frac{\delta H_{2 n+2}}{\delta u}\right)\right|_{u=0}=\partial \frac{1}{2}\left(\frac{\delta \bar{H}_{2 n+3}}{\delta h}\right) \\
& =\left(h \partial+\partial h+\frac{1}{2} \partial^{3}\right)\left(\frac{\delta \bar{H}_{2 n+1}}{\delta h}\right),
\end{aligned}
$$

which is eq. (2.68).

Similar results can be derived for the nonabelian integrable systems of the preceding subsection.

Theorem 2.3. (i) The $\mathfrak{g}-m D W W$ system

$$
\left(\begin{array}{l}
\mathbf{U} \\
\overline{\mathbf{V}}
\end{array}\right)_{t}=\left(\begin{array}{cc}
-2[\mathbf{U},]+\partial \mathbf{1} & \mathbf{0} \\
\mathbf{0} & -[\overline{\mathbf{V}},]-\frac{1}{2} \partial \mathbf{1}
\end{array}\right)\left(\begin{array}{l}
\delta / \delta \mathbf{U} \\
\delta / \delta \overline{\mathbf{V}}
\end{array}\right) \Phi_{(1)}\left(H_{2 n+1}\right)
$$


has the invariant submanifold defined by

$$
\mathbf{U}=\mathbf{0}
$$

on which it becomes

$$
\overline{\mathbf{V}}_{, t}=\left(-[\overline{\mathbf{V}}, \quad]-\frac{1}{2} \partial \mathbf{1}\right) \frac{\delta}{\delta \overline{\mathbf{V}}}\left(\Phi_{(1)}\left(\bar{H}_{2 n+1}\right)\right) .
$$

(ii) The map $\Phi_{(1)}$ on the submanifold $\{\mathbf{U}=\mathbf{0}\}$ :

$$
\bar{\Phi}_{(1)}(h):=\left.\Phi_{(1)}(h)\right|_{\mathbf{U}=\mathbf{0}}=\boldsymbol{\theta}^{t} \overline{\mathbf{V}}^{\prime}-\overline{\mathbf{V}}^{t} \overline{\mathbf{V}}
$$

is a Hamiltonian map into the second Hamiltonian structure of the $K d V$ hierarchy (2.68):

$$
h_{t}=\left(h \partial+\partial h+\frac{1}{2} \partial^{3}\right)\left(\frac{\delta \bar{H}_{2 n+1}}{\delta h}\right) .
$$

Proof. (i) By formulae (2.50) and (2.46),

$$
\begin{aligned}
\left.\frac{\delta \Phi_{(1)}\left(H_{2 n+1}\right)}{\delta \mathbf{U}}\right|_{\mathbf{U}=\mathbf{0}} & =\left.\boldsymbol{\theta}^{t} \Phi_{(1)}\left(\frac{\delta H_{2 n+1}}{\delta u}-\frac{1}{2} \partial\left(\frac{\delta H_{2 n+1}}{\delta h}\right)\right)\right|_{u=0} \\
{[\text { by }(2.70 \mathrm{~b})] } & =\boldsymbol{\theta}^{t} \Phi_{(1)}\left(\frac{1}{2} \frac{\delta \bar{H}_{2 n+2}}{\delta h}\right) \\
{[\text { by }(2.70 \mathrm{a})] } & =\boldsymbol{\theta}^{t} \Phi_{(1)}(0)=\mathbf{0} .
\end{aligned}
$$

Hence, from (2.74),

$$
\left.\mathbf{U}_{t}\right|_{\mathbf{U}=\mathbf{0}}=\partial\left(\left.\frac{\delta \Phi_{(1)}\left(H_{2 n+1}\right)}{\delta \mathbf{U}}\right|_{\mathbf{U}=\mathbf{0}}\right)=\mathbf{0}
$$

so that $\mathbf{U}=\mathbf{0}$ indeed defines an invariant submanifold.

(ii) From formulae (2.50) and (2.52) we have

$$
\frac{\delta \Phi_{(1)}\left(\bar{H}_{2 n+1}\right)}{\delta \overline{\mathbf{V}}}=\left.\frac{\delta \Phi_{1}\left(H_{2 n+1}\right)}{\delta \overline{\mathbf{V}}}\right|_{\mathbf{U}=\mathbf{0}}=-(\boldsymbol{\theta} \partial+2 \overline{\mathbf{V}}) \Phi_{(1)}\left(\frac{\delta \bar{H}_{2 n+1}}{\delta h}\right),
$$

since letting $\mathbf{U}$ vanish does not interfere with taking variational derivatives with respect to $\overline{\mathbf{V}}$. Hence, from the second row of formula (2.74) we conclude that

$$
\left.\overline{\mathbf{V}}_{t}\right|_{\mathbf{U}=\mathbf{0}}=\left(-[\overline{\mathbf{V}},]-\frac{1}{2} \partial \mathbf{1}\right)\left(\frac{\delta \Phi_{(1)}\left(\bar{H}_{2 n+1}\right)}{\delta \overline{\mathbf{V}}}\right)
$$


which is (2.76). Then a computation similar to the one at the end of subsection 2a shows that

$$
\bar{\Phi}_{(1)}\left(h \partial+\partial h+\frac{1}{2} \partial^{3}\right)=D\left[\bar{\Phi}_{(1)}(h)\right]\left(-[\overline{\mathbf{V}},]-\frac{1}{2} \partial \mathbf{1}\right)\left\{D\left[\bar{\Phi}_{(1)}(h)\right]\right\}^{\dagger} .
$$

It follows from Theorem 2.2 that condition (2.75) picks out the flows of eq. (2.76), which are the $\mathfrak{g}-\mathrm{mKdV}$ flows constructed in [Ku2].

The problem of specialization is slightly different for the $\mathfrak{g}-\mathrm{m}^{2} \mathrm{DWW}$ hierarchy, defined by

$$
\left(\begin{array}{l}
\mathbf{U} \\
\mathbf{V}
\end{array}\right)_{t}=\left(\begin{array}{cc}
\mathbf{0} & -[\mathbf{U},]+\partial \mathbf{1} \\
-[\mathbf{U},]+\partial \mathbf{1} & -[\mathbf{V},]
\end{array}\right)\left(\begin{array}{c}
\delta / \delta \mathbf{U} \\
\delta / \delta \mathbf{V}
\end{array}\right) \Phi_{(0)}\left(\mathcal{H}_{m}\right)
$$

To begin with, the hierarchy related to it by the map $\Phi_{(0)}$ :

$$
\left(\begin{array}{l}
u \\
h
\end{array}\right)_{t}=\left(\begin{array}{cc}
0 & \partial u-\partial^{2} \\
u \partial+\partial^{2} & h \partial+\partial h
\end{array}\right)\left(\begin{array}{c}
\delta / \delta u \\
\delta / \delta h
\end{array}\right)\left(\mathcal{H}_{m}\right)
$$

has an invariant submanifold defined by

$$
u+2 h=0
$$

for all $m \equiv 1(\bmod 2)$. This follows from the equality

$$
\frac{\delta \mathcal{H}_{2 n+1}}{\delta h}=2 \frac{\delta \mathcal{H}_{2 n+1}}{\delta u} \quad \text { on } \quad\{u+2 h=0\} .
$$

(see $[\mathbf{R}])$. Fixing $n$ and defining

$$
\mathcal{X}_{n}:=\left.\frac{\delta \mathcal{H}_{2 n+1}}{\delta u}\right|_{u+2 h=0}
$$

we obtain from formula (2.62) that on the submanifold defined by

$$
\Gamma:=\boldsymbol{\theta}^{t}\left(\mathbf{U}+2 \mathbf{V}^{\prime}\right)+2 \mathbf{U}^{t} \mathbf{V}=0
$$


which is the image under the homomorphism $\Phi_{(0)}(2.43 \mathrm{a}, \mathrm{b})$ of the invariant submanifold determined by $u+2 h=0$, we have

$$
\begin{aligned}
& \left.\frac{\delta \Phi_{(0)}\left(\mathcal{H}_{2 n+1}\right)}{\delta \mathbf{U}}\right|_{\Gamma=0}=\mathcal{X}_{n}(\boldsymbol{\theta}+2 \mathbf{V}) \\
& \left.\frac{\delta \Phi_{(0)}\left(\mathcal{H}_{2 n+1}\right)}{\delta \mathbf{V}}\right|_{\Gamma=0}=2 \mathcal{X}_{n} \mathbf{U}-2 \mathcal{X}_{n}^{\prime} \boldsymbol{\theta}
\end{aligned}
$$

Hence, on the submanifold defined by $\Gamma=0$, our $\mathfrak{g}-\mathrm{m}^{2} \mathrm{DWW}$ system $(2.83)$ becomes

$$
\begin{aligned}
& \mathbf{U}_{t}=2 \mathcal{X}_{n}^{\prime}[\mathbf{U}, \boldsymbol{\theta}]+\left(2 \mathcal{X}_{n} \mathbf{U}-2 \mathcal{X}_{n}^{\prime} \boldsymbol{\theta}\right)^{\prime} \\
& \mathbf{V}_{t}=\mathcal{X}_{n}[\boldsymbol{\theta}, \mathbf{U}]+2 \mathcal{X}_{n}^{\prime}[\mathbf{V}, \boldsymbol{\theta}]+\left[\mathcal{X}_{n}(\boldsymbol{\theta}+2 \mathbf{V})\right]^{\prime}
\end{aligned}
$$

Proposition 2.4. The flow determined by (2.90) leaves the submanifold defined by $\Gamma=0$ invariant.

Proof. From $(2.90 \mathrm{a}, \mathrm{b})$, we obtain

$$
\Gamma_{t}=2 \mathcal{X}_{n} \Gamma^{\prime}+4 \mathcal{X}_{n}^{\prime} \Gamma
$$

Remark 2.3. The constraint $\Gamma=0$ in (2.88) is differential and cannot be resolved algebraically, except in the classical case when $\mathfrak{g}=\mathbb{R}^{1}$ and $\boldsymbol{\theta}=1$, resulting in the formula

$$
U=-(1+2 V)^{-1} V^{\prime}=\left[-\frac{1}{2} \ln (1+2 V)\right]^{\prime},
$$

so that one is dealing with the potential mKdV hierarchy.

\section{2d. Integrable Systems on $T^{*} \widetilde{G}$}

From formula $(1.10 \mathrm{~d})$ for the vector field $X_{H}$ of the Hamiltonian $H(g, \mu)$, the equations of motion may be expressed as

$$
\left(\begin{array}{c}
\dot{g} \\
\dot{\mu}
\end{array}\right)=B^{k}\left(\begin{array}{c}
\frac{\delta H}{\delta g} \\
\frac{\delta H}{\delta \mu}
\end{array}\right),
$$


where the Hamiltonian matrix $B^{k}$ is

$$
B^{k}=\left(\begin{array}{cc}
0 & L_{g *} \\
-R_{g}^{*} & {\left[\mu+k g^{-1} g^{\prime}, .\right]+k \partial}
\end{array}\right),
$$

and the notation $L_{g *}$ and $R_{g}^{*}$ signifies left (resp. right) multiplication of the $\mathfrak{g}$ element $\frac{\delta H}{\delta \mu}$ (resp. the $T_{g}^{*} \widetilde{G}$ element $\frac{\delta H}{\delta g}$ ) by $g$. The integrable commuting hierarchies constructed in the previous subsections generate commuting hierarchies on $T^{*} \widetilde{G}$ by pulling back those on $\operatorname{diff}_{1(\alpha)}^{\wedge *}$ under the Poisson maps $(1.76,1.108)$ of Section 1.

For the case $(\alpha)=(0)$, the commuting system of Hamiltonians is generated by $\left\{\left(\hat{J}^{L A}\right)^{*} \phi_{(0)}\left(\mathcal{H}_{n}\right)\right\}$. For $n=1$, by formula $(2.58)$, we have $\Phi_{(0)}\left(\frac{1}{2} \mathcal{H}_{1}\right)=\Phi_{(0)}(h) \sim \mathbf{U}^{t} \mathbf{V}$. Since, by formula (1.46),

$$
\begin{aligned}
& \left(\hat{J}^{L A}\right)^{*}(\mathbf{U})=g^{\prime} g^{-1} \\
& \left(\hat{J}^{L A}\right)^{*}(\mathbf{V})=g \mu g^{-1},
\end{aligned}
$$

we find that

$$
\begin{aligned}
\frac{\delta}{\delta g}\left(\hat{J}^{L A}\right)^{*}\left(\mathbf{U}^{t} \mathbf{V}\right) & =\left(-\mu^{\prime}+\left[\mu, g^{-1} g^{\prime}\right]\right) g^{-1} \\
\frac{\delta}{\delta \mu}\left(\hat{J}^{L A}\right)^{*}\left(\mathbf{U}^{t} \mathbf{V}\right) & =g^{-1} g^{\prime},
\end{aligned}
$$

and the equations of motion (2.93), (2.94) yield

$$
\left(\begin{array}{l}
\dot{g} \\
\dot{\mu}
\end{array}\right)=\left(\begin{array}{c}
g^{\prime} \\
\mu^{\prime}
\end{array}\right),
$$

which is just the $\sigma$-shift, as expected. For all the other Hamiltonians $\mathcal{H}_{n}$, we let:

$$
\delta \Phi_{(0)}\left(\mathcal{H}_{n}\right)=: \widetilde{a}_{n}^{t} \delta \mathbf{U}+\widetilde{b}_{n}^{t} \delta \mathbf{V} .
$$

Thus, e.g., $\widetilde{a}_{2}$ and $\widetilde{b}_{2}$ are given by the components of the vector (2.63). Denoting

$$
a_{n}:=\left(\hat{J}^{L A}\right)^{*}\left(\widetilde{a}_{n}\right), \quad b_{n}:=\left(\hat{J}^{L A}\right)^{*}\left(\widetilde{b}_{n}\right),
$$

we find that

$$
\begin{aligned}
\frac{\delta}{\delta g}\left(\hat{J}^{L A}\right)^{*} \Phi_{(0)}\left(\mathcal{H}_{n}\right) & =g^{-1}\left[g \mu g^{-1}, b_{n}\right]-\left(g^{-1} a_{n}\right)^{\prime}-g^{-1} a_{n} g^{\prime} g^{-1} \\
\frac{\delta}{\delta \mu}\left(\hat{J}^{L A}\right)^{*} \Phi_{(0)}\left(\mathcal{H}_{n}\right) & =g^{-1} b_{n} g,
\end{aligned}
$$


so that the equations of motion on $T^{*} \widetilde{G}$ for the Hamiltonian $\left(\hat{J}^{L A}\right)^{*} \Phi_{(0)}\left(\mathcal{H}_{n}\right)$ are

$$
\begin{aligned}
& \dot{g}=b_{n} g \\
& \dot{\mu}=-\left(g^{-1} a_{n} g\right)^{\prime} .
\end{aligned}
$$

Next, consider the case $(\alpha)=(1)$. Here we get the infinite commuting hierarchy $\left\{\left(\hat{J}_{k}^{L R}\right)^{*} \Phi_{(1)}\left(H_{n}\right)\right\}$, where $\hat{J}_{k}^{L R}$ is given by formula (1.23):

$$
\begin{aligned}
& \left(\hat{J}^{L R}\right)^{*}(\overline{\mathbf{U}})=g \mu g^{-1}, \quad \overline{\mathbf{U}}:=\frac{1}{2} \mathbf{U} \\
& \left(\hat{J}^{L R}\right)^{*}(\overline{\mathbf{V}})=-\mu+k g^{-1} g^{\prime}, \quad k=\frac{1}{2} .
\end{aligned}
$$

For the Hamiltonian $H_{1}=h$, we have from $(2.42 \mathrm{~b})$ that $\Phi_{(1)}\left(H_{1}\right) \sim \overline{\mathbf{U}}^{t} \overline{\mathbf{U}}-\overline{\mathbf{V}}^{t} \overline{\mathbf{V}}$, so that

$$
\begin{gathered}
\delta \Phi_{(1)}\left(H_{n}\right)=: \widetilde{a}_{n}^{t} \delta \overline{\mathbf{U}}+\widetilde{b}_{n}^{t} \delta \overline{\mathbf{V}} \\
\widetilde{a}_{1}=2 \overline{\mathbf{U}}, \quad \widetilde{b}_{1}=-2 \overline{\mathbf{V}} .
\end{gathered}
$$

Denoting

$$
\widehat{a}_{n}:=\left(\hat{J}^{L R}\right)^{*}\left(\widetilde{a}_{n}\right), \quad \widehat{b}_{n}:=\left(\hat{J}^{L R}\right)^{*}\left(\widetilde{b}_{n}\right),
$$

from formulae $(2.102 \mathrm{a}, \mathrm{b})$ we get

$$
\begin{aligned}
& \frac{\delta}{\delta g}\left(\hat{J}_{k}^{L R}\right)^{*} \Phi_{(1)}\left(H_{n}\right)=g^{-1}\left[g \mu g^{-1}, \widehat{a}_{n}\right]-k\left(\widehat{b}_{n}^{\prime}+\left[g^{-1} g^{\prime}, \widehat{b}_{n}\right]\right) g^{-1} \\
& \frac{\delta}{\delta \mu}\left(\hat{J}_{k}^{L R}\right)^{*} \Phi_{(1)}\left(H_{n}\right)=-\widehat{b}_{n}+g^{-1} \widehat{a}_{n} g
\end{aligned}
$$

and formula (2.93) yields the following Hamiltonian equations on $T^{*} \widetilde{G}$ for the Hamiltonian $\left(\hat{J}_{k}^{L R}\right)^{*} \Phi_{(1)}\left(H_{n}\right)$ :

$$
\begin{aligned}
& \dot{g}=\widehat{a}_{n} g-g \widehat{b}_{n} \\
& \dot{\mu}=k\left(g^{-1} \widehat{a}_{n} g\right)^{\prime}+\left[\widehat{b}_{n}, \mu\right]+k\left[g^{-1} g^{\prime}, g^{-1} \widehat{a}_{n} g\right], \quad k=\frac{1}{2} .
\end{aligned}
$$

In particular for $n=1$, substituting formulae (2.104) and (2.102a,b) in (2.107a,b), we get the $\sigma$-shift flow, as expected. 


\section{2e. WZW Model [Wi, FT, H]}

For the 3-parameter family of Hamiltonians on $T^{*} \widetilde{G}$

$$
H=\int\left[\alpha_{1}\langle\mu, \mu\rangle+\alpha_{2}\left\langle\alpha_{2}, g^{-1} g^{\prime}\right\rangle+\alpha_{3}\left\langle g^{-1} g^{\prime}, g^{-1} g^{\prime}\right\rangle\right] d \sigma
$$

we have

$$
\begin{aligned}
& \frac{\delta H}{\delta g}=-\left\{\left(2 \alpha_{3} g^{-1} g^{\prime}+\alpha_{2} \mu\right)^{\prime}+\left[g^{-1} g^{\prime}, \alpha_{2} \mu\right]\right\} g^{-1} \\
& \frac{\delta H}{\delta \mu}=2 \alpha_{1} \mu+\alpha_{2} g^{-1} g^{\prime}
\end{aligned}
$$

The corresponding equations of motion, by formula (2.93), are therefore

$$
\begin{aligned}
& \dot{g}=2 \alpha_{1} g \mu+\alpha_{2} g^{\prime} \\
& \dot{\mu}=\left\{\left(2 \alpha_{3}+\alpha_{2} k\right) g^{-1} g^{\prime}+\left(\alpha_{2}+2 \alpha_{1} k\right) \mu\right\}^{\prime}+2 \alpha_{1} k\left[g^{-1} g^{\prime}, \mu\right] .
\end{aligned}
$$

Eliminating the momentum $\mu$ from these equations gives the second order system

$$
\frac{1}{2 \alpha_{1}}\left(g^{-1} \dot{g}\right)^{\cdot}=\left(2 \alpha_{3}+\frac{k}{2} \alpha_{2}\right)\left(g^{-1} g^{\prime}\right)^{\prime}+\frac{k}{2}\left[g^{-1} \dot{g}, g^{-1} g^{\prime}\right]
$$

Hence, choosing

$$
\alpha_{1}=\frac{\varepsilon}{k}, \quad \alpha_{2}=-\varepsilon, \quad \alpha_{3}=\frac{\varepsilon k}{2}, \quad \varepsilon= \pm 1,
$$

we obtain the standard WZW system [Wi]

$$
\left(g^{-1} \dot{g}\right)^{\cdot}-\left(g^{-1} g^{\prime}\right)^{\prime}=\varepsilon\left[g^{-1} \dot{g}, g^{-1} g^{\prime}\right]
$$

The Hamiltonian $H$ in (2.108) for these choices of the parameters $\alpha_{1}, \alpha_{2}, \alpha_{3}$ is:

$$
H_{W Z W}=\frac{\varepsilon}{2 k} \int\left[\langle\mu, \mu\rangle+\left\langle-\mu+k g^{-1} g^{\prime},-\mu+k g^{-1} g^{\prime}\right\rangle\right] d \sigma
$$

which by formulae $(2.102 \mathrm{a}, \mathrm{b})$, with $k=\frac{1}{2}$ and $\varepsilon=1$, is

$$
\left(\widehat{J}^{d}\right)^{*}\left(\int\left[\frac{\mathbf{U}^{t} \mathbf{U}}{4}+\overline{\mathbf{V}}^{t} \overline{\mathbf{V}}\right] d \sigma\right)
$$


i.e., the pull back of the Hamiltonian

$$
\left.\widehat{H}_{W Z W}=\int\left[\frac{\mathbf{U}^{t} \mathbf{U}}{4}+\overline{\mathbf{V}}^{t} \overline{\mathbf{V}}\right)\right] d \sigma=\int\left[\left(\overline{\mathbf{U}}^{t} \overline{\mathbf{U}}^{t} \overline{\mathbf{V}}^{t} \overline{\mathbf{V}}\right] d \sigma\right.
$$

from $(\widetilde{\mathfrak{g}} \oplus \widetilde{\mathfrak{g}})^{\wedge *}$. The corresponding equations of motion on $(\widetilde{\mathfrak{g}} \oplus \widetilde{\mathfrak{g}})^{\wedge *}$ are, by formula (2.33), just the usual left and right translational modes

$$
\begin{aligned}
& \dot{\overline{\mathbf{U}}}=\overline{\mathbf{U}}^{\prime} \\
& \dot{\overline{\mathbf{V}}}=-\overline{\mathbf{V}}^{\prime} .
\end{aligned}
$$

Since the system $(2.117 \mathrm{a}, \mathrm{b})$ has integrals of the form

$$
F_{1}(\overline{\mathbf{U}})+F_{2}(\overline{\mathbf{V}})
$$

with arbitrary $\sigma$-independent Hamiltonians $F_{1}$ and $F_{2}$, the WZW system (2.109) has an infinite number of integrals of the form

$$
\int\left[F_{1}\left(g \mu g^{-1}\right)+F_{2}\left(-\mu+\frac{1}{2} g^{-1} g^{\prime}\right)\right] d \sigma .
$$

These integrals commute, for distinct pairs $\left(F_{1}, F_{2}\right)$ and $\left(\tilde{F}_{1}, \tilde{F}_{2}\right)$, if $\left(F_{1}, \tilde{F}_{1}\right)$ and $\left(F_{2}, \tilde{F}_{2}\right)$ separately do. Thus, we may choose them from any two commuting hierarchies on $\widetilde{\mathfrak{g}}^{\wedge *}$. There is a distinguished such hierarchy; namely, the nonabelian $\mathrm{mKdV}$ hierarchy [Ku2], obtained by pulling back the KdV integrals under the map $\Phi$ of (2.27) with an arbitrary fixed $\boldsymbol{\theta}$ of length 1 :

$$
\Phi\left(h_{n}\right)=: h_{n}^{\boldsymbol{\theta}} .
$$

Hence, we have the doubly infinite commuting system on $(\widetilde{\mathfrak{g}} \oplus \widetilde{\mathfrak{g}})^{\wedge *}$ consisting of Hamiltonians of the form $\left\{h_{n}^{\boldsymbol{\theta}_{1}}(\overline{\mathbf{U}})+h_{m}^{\boldsymbol{\theta}_{2}}(\overline{\mathbf{V}})\right\}$. This, in turn, furnishes the KdVgenerated doubly infinite commuting hierarchy of Hamiltonians on $T^{*} \widetilde{G}$ of the form $\left\{h_{n}^{\boldsymbol{\theta}_{1}}\left(g \mu g^{-1}\right)+h_{m}^{\boldsymbol{\theta}_{2}}\left(-\mu+\frac{1}{2} g^{-1} g^{\prime}\right)\right\}$, which include the generator of the left-right translational flow giving the WZW model as its first element. 


\section{REFERENCES}

[AHP] Adams, M.R., Harnad, J. and Previato, E., "Isospectral Hamiltonian Flows in Finite and Infinite Dimensions I. Generalised Moser Systems and Moment Maps into Loop Algebras", Commun. Math. Phys. 117, 451-500 (1988); Adams, M.R., Harnad, J. and Hurtubise, J., "Isospectral Hamiltonian Flows in Finite and Infinite Dimensions II. Integration of Flows", Commun. Math. Phys. 134, 555-585 (1990).

[DJKM] Date, E., Kashiwara, M., Jimbo, M. and Miwa, T., "Transformation Groups for Soliton Equations", I. Proc. Jap. Acad. 57A, 342-347 (1981); II. ibid., 387-392 (1981); III. J. Phys. Soc Japan, 50, 3806-3812 (1981); VI. ibid. 50 3813-3818 (1981); V. Publ. Res. Inst. Math. Sci. 18, 1077-1110 (1982); ibid., 1111-1119 (1982); IV. Physica 4D, 343-365 (1982).

[DS] Drinfeld, V. G. and Sokolov, V. V., "Lie algebras and Equations of Korteweg - De Vries Type", Jour. Sov. Math. 30 1975-2036 (1985); "Equations of Korteweg-De Vries type and Simple Lie Algebras", Soviet Math. Dokl. 23, 457-462 (1981).

[FNR] Flaschka, H., Newll, A.C. and Ratiu, T., "Kac-Moody Algebras and Soliton Equations II. Lax

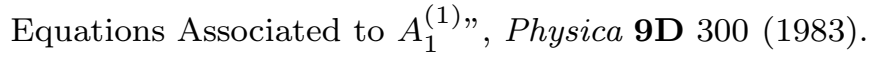

[FT] Faddeev, L.D. and Takhtajan, L.A., Hamiltonian Methods in the Theory of Solitons, Part II, Ch.1.5, Springer-Verlag, Heidelberg (1987).

[H] Harnad, J., "Constrained Hamiltonian systems on Lie Groups, Moment Map Reductions and Central Extensions", preprint CRM (1990).

[HK1] Harnad, J. and Kupershmidt, B. A., "Twisted Diff $S^{1}$-action on Loop Groups and Representations of the Virasoro Algebra", Lett. Math. Phys. 19, 277-284 (1990).

[HK2] Harnad, J. and Kupershmidt, B. A., "Hamiltonian Group Actions on Superloop Spaces", Commun. Math. Phys. 132, 315-347 (1990).

[Ku1] Kupershmidt, B. A., "Mathematics of Dispersive Water Waves", Commun. Math. Phys. 99, 51-73 (1985).

[Ku2] Kupershmidt, B. A., "Modified Korteweg-de Vries Equations on Euclidean Lie Algebras", Int. J. Mod. Phys. 3, 853-861 (1989).

[PS] Pressley, A. and Segal, G., Loop Groups, Clarendon Press, Oxford (1986).

[RS] Reiman, A. G. and Semenov-Tian-Shansky, M. A., "Reductions of Hamiltonian Systems, Affine Lie Algebras and Lax Equations I and II", Invent. Math. 54, 81-100 (1979); ibid. 63, 423-432 (1981).

[R] Razboinick, S. I., "Vector Extensions of Modified Water Wave Equations", Phys. Lett. 119A, 283-286 (1986).

[SW] Segal, G. and Wilson, G., "Loop Groups and Equations of KdV Type", Publ. Math. IHES 61, 5-65 (1985).

[W1] Wilson, G., "Habillage et fonctions $\tau$ ", C. R. Acad. Sci. Paris, Sér. I 299, 587-590 (1984).

[W2] Wilson, G., "On the Quasi-Hamiltonian Formalism of the KdV Equation", Phys. Lett. 132A, 445-450 (1988).

[Wi] Witten, E., "Nonabelian Bosonization in Two Dimensions", Commun. Math. Phys. 92, 452-472 (1984). 